\title{
Reducing Math Anxiety in School Children: A Systematic Review of Intervention Research
}

\author{
Miriam Balt ${ }^{1 *}$, Moritz Börnert-Ringleb ${ }^{2}$ and Lars Orbach ${ }^{3}$ \\ ${ }^{1}$ Institute of Educational Research, University of Wuppertal, Wuppertal, Germany, ${ }^{2}$ Institute of Special Education, Leibniz \\ University Hannover, Hanover, Germany, ${ }^{3}$ Department of Psychology, Federal University of Minas Gerais, Belo Horizonte, Brazil
}

Recent studies indicate that math anxiety (MA) can already be found in school-aged children. As early MA depicts a potential risk for developing severe mathematical difficulties and impede the socio-emotional development of children, distinct knowledge about how to reduce MA in school-aged children is of particular importance. Therefore, the goal of this systematic review is to summarize the existing body of research on MA interventions for children by identifying the approaches, designs, and characteristics as well as the effects of the interventions.

Keywords: math anxiety, intervention, review, school, children

OPEN ACCESS

Edited by:

Riikka Mononen,

University of Oulu, Finland

Reviewed by:

Florence Claude Gabriel,

University of South Australia, Australia

Brenda RJ Jansen,

University of Amsterdam, Netherlands

${ }^{*}$ Correspondence:

Miriam Balt

mbalt@uni-wuppertal.de

Specialty section:

This article was submitted to Educational Psychology, a section of the journal

Frontiers in Education

Received: 20 October 2021 Accepted: 06 January 2022 Published: 03 February 2022

Citation:

Balt $M$, Börnert-Ringleb $M$ and Orbach L (2022) Reducing Math

Anxiety in School Children: A

Systematic Review of

Intervention Research.

Front. Educ. 7:798516.

doi: 10.3389/feduc.2022.798516

\section{INTRODUCTION}

In the last decade, a considerable amount of research focused on math anxiety (MA). Ramirez et al. (2018) sum up results of across 65 countries that participated in the 2012 PISA survey and highlight that " $33 \%$ of 15 -year-old students, on average, reported feeling helpless when solving math problems" (p.146). In accordance with the high prevalence in this age group, the majority of existing studies addressed MA in adolescents and young adults. However, more recent research described MA as early as in primary school children (Ramirez et al., 2013; Cargnelutti et al., 2017; Gunderson et al., 2018; Sorvo et al., 2019; Primi et al., 2020) and highlighted negative impacts of early MA on their short- and long-term development and performance in mathematics (Sorvo et al., 2017; Namkung et al., 2019; Zhang et al., 2019; Barroso et al., 2021). However, until now little attention has been paid to the investigation of interventions aiming at the reduction of MA in children (Passolunghi et al., 2020). The paper at hand aims to systematically review the existing literature on interventions and approaches that target to reduce MA in school-aged children.

\section{THEORETICAL BACKGROUND}

\subsection{Definition of MA}

MA can generally be defined as an "anxiety that interferes with manipulation of numbers and the solving of mathematical problems in a wide variety of ordinary life and academic situations" (Richardson and Suinn, 1972, p.551). There is, however, no consensus on the exact operationalization of MA in the field (e.g., Dowker et al., 2016). One important step towards a more precise definition and operationalization of MA is offered by the distinction of MA into trait and state anxiety. According to Spielberger (1972) trait anxiety refers to a relatively enduring individual disposition to feel anxious, whereas state anxiety refers to temporary and situational feelings of anxiety. Current MA studies either assess anxiety in math-related situations using 
hypothetical/retrospective questions (e.g., "How anxious would you feel if ... ") or assess anxiety about failure in math (e.g., "How worried are you if you have problems with ..."). The first type of question allows assumptions about state-like MA as not administered within the actual situation, the second type of question provides indications about trait MA (Sorvo et al., 2017; Orbach et al., 2019). Considering empirical discrepancies between MA self-reports (see questions above) and real-time assessments (Bieg, 2013), nowadays more studies apply questionnaires assessing state-MA within the actual mathematical situation (e.g., Vanbecelaere et al., 2021).

\subsection{Explaining MA in Children}

The development of MA and its relation to math performance has been investigated in only a few longitudinal studies (Sorvo et al., 2019). According to these studies different etiological pathways have been suggested (Carey et al., 2017; Sorvo et al., 2019) and it has been assumed that the MA-performance link is bidirectional (Carey et al., 2016; Foley et al., 2017). In other words, MA can be considered as both the cause and the outcome of poor math performance (Young et al., 2012).

Accordingly, MA could be elicited or increased over time because of math difficulties that often originate in early school years (Ramirez et al., 2018). Ramirez et al. (2018) define this as reduced competency account and explain this link in two ways: A first explanation might be seen in lower numerical/spatial abilities which lead to underperformance in math and consequently to MA. Barroso et al. (2021) describe this association as the "deficit model" of MA (p.136). Ramirez et al. (2018) further summarize, that a second explanation could be seen in avoidance behavior that amplifies the development of math difficulties and consequent MA. In line with this, Ashcraft and Moore (2009) state that "avoidance of math is an overriding characteristic of math-anxious individuals" (p. 201). Therefore, experiencing math difficulties might cause a "vicious circle" (Dowker et al., 2016) in which students avoid math-related situations leading to fewer opportunities to improve their math skills. Ramirez et al. (2018) consequently argue, that according to the assumption that MA may be the outcome of poor math performance, “interventions that aim to improve students' math skills may be effective" to reduce MA (p. 156). Consequently, recent studies suggest a positive effect of mathematical interventions (MI) on MA in school children (e.g., Supekar et al., 2015; Passolunghi et al., 2020; Vanbecelaere et al., 2020).

Performance-inhibiting effects might, however, also be caused by MA. Such types of MA might be originally developed from environmental factors (e.g., adult role models: Casad et al., 2015; Lin et al., 2017) and genetic dispositions (Wang et al., 2014; Malanchini et al., 2017). Such MA-related impacts on mathematical performance might be explained by the disruption of executive function processes and working memory (disruption account; Ramirez et al., 2018). This disruption may be caused by math-related worries (e.g., negative thoughts and rumination about one's abilities or the consequences of failure). As a result, MA-evoking situations interfere with available cognitive resources (e.g., working memory) (e.g., Ramirez et al., 2013; Pizzie et al., 2020).
Therefore, less resources are available for task-related problem-solving processes (e.g., arithmetical strategies). This might lead children either to switch to less sophisticated strategies (e.g., production deficiencies) or apply advanced strategies unsuccessfully (e.g., utilization deficiencies; Miller and Seier, 1994), both approaches leading to poorer performances. Barroso et al. (2021) summarize such links under the "processing efficiency theory" of MA (p.136). The links between MA and performance might additionally be influenced by the complexity of math tasks that children have to solve and the presence of time pressure. Studies using math assessments including more complex tasks show stronger MAperformance links (Namkung et al., 2019; Zhang et al., 2019). Another stress-evoking factor might be seen in time pressure, as it seems to affect the arousal of children (Caviola et al., 2017a; Orbach et al., 2020). According to the assumption of a disruption of executive functions caused by math-related worries, cognitivebehavioral interventions (CBI) may help children to deal with maladaptive thoughts that e.g., attribute poor math grades to a lack of ability. Recent studies suggest a positive effect of CBI on MA in school children (e.g., Passolunghi et al., 2020).

\subsection{Reducing MA in Children}

With regard to the described manifold link between MA and mathematical performance, it becomes clear that reducing symptoms of MA might be a relevant approach in supporting children's mathematical development (Passolunghi et al., 2020). At the same time, the multiple explanations of the link between MA and mathematical performance might serve as a diverse foundation for designing appropriate interventional activities (e.g., addressing numerical/spatial abilities, executive functions, math self-concept). Previous work highlighted that the existing body of research can be subsumed into interventions that primarily target mathematical abilities as well as into cognitive-behavioral interventions that target anxiety related cognitions (Dowker et al., 2016). Both directions can thereby be interpreted with regard to the described differential links between MA and mathematical performance.

As described, MI might be of particular relevance in light of the described reduced competency account (Ramirez et al., 2018). They aim to break the vicious circle of MA and performance by promoting mathematical performance and thereby increasing math self-concept as well as decreasing MA. In line with this argument Dowker et al. (2016) propose that "interventions for children with mathematical difficulties may go some way toward preventing a vicious spiral, where mathematical difficulties cause anxiety, which causes further difficulties with mathematics" (p. 10). Similarly, math trainings moreover depict exposure interventions. Accordingly, Ramirez et al. (2018) argue that "the avoidance framework under the Reduced Competency Account states that avoidance tendencies may be responsible for the deficits in development (and explains why increased exposure is an effective solution)" (p. 156).

The effects of CBI can be mainly explained with regard to the described disruption account (Ramirez et al., 2018). Accordingly, CBI might decline the potential impact of 
anxiety-related cognitive processes and by that means improve mathematical performance. Dowker et al. (2016) as well as Ramirez et al. (2018) both highlight the potential impact of $\mathrm{CBI}$ such as re-appraisal and expressive writing on MA.

\section{OBJECTIVE OF THE STUDY AND RESEARCH QUESTIONS}

Most of the existing body of research on MA and MA interventions appears to focus on older adolescents and adults, as MA has been previously associated with more complex mathematics. At the same time, MA could already be observed in school-aged children and might be associated with early mathematical functioning and numeracy. Therefore, early identification and intervention of MA seems to be of high relevance to prevent negative developmental outcomes. As research on early MA interventions is limited, the exact conditions and characteristics of successful interventions in school-aged children remain unclear. To our knowledge, no existing work has summarized the existing evidence on the interventional approaches that target MA in childhood. Therefore, the objective of this study is to give an overview of interventional approaches in addressing MA in children and adolescents and to highlight potential characteristics of effective interventions. The study is guided by the following research questions:

1) What are the approaches, designs, and characteristics (e.g., setting, duration) of existing interventions aiming at the reduction of $\mathrm{MA}$ in school children?

2) What are the effects of these existing interventions?

Answers to these questions might contribute to the field of MA intervention research, as they might serve as a foundation and orientation for future intervention studies aiming at improving children's emotional well-being and academic development in schools, especially regarding mathematics.

\section{METHODS}

As MA has been addressed in previous research, we aim to identify characteristics of effective interventions based on the existing body of research. Therefore, we conduct a systematic (scoping) review. Thereby, we will describe the main findings of the included studies and highlight specific components using a narrative approach.

\subsection{Search Procedure}

To identify all relevant studies, we used a two-step approach. In a first step we conducted a systematic search in the most widely used electronic databases in psychological and educational research. Therefore, we focused on the databases PsycINFO and PubPsych. PubPsych is a multilingual database that includes entries from additional databases, such as PSYNDEX,
MEDLINE and ERIC (Educational Resources Information Center). We used the descriptors: math (ematics) anxiety AND intervention OR treatment OR therapy OR program OR training OR tutoring OR support OR strategies OR best practice, AND alleviation as well as its synonyms reduction OR decrease OR remediation. Additionally, a German translation of the descriptors was used. To prevent the exclusion of relevant studies at an early stage no filters were used except the exclusion of dissertations as full texts are often difficult to access. We additionally identified studies by hand search, i.e., visually scanning reference lists from relevant studies or theoretical papers. The literature search was conducted in July 2020 and October 2021.

\subsubsection{Inclusion and Exclusion Criteria}

Studies were eligible for the systematic review if they met all the following inclusion criteria:

- Participants received intervention or a combination of interventions.

- Participants were assessed with a quantitative and/or qualitative measure of MA.

- Participants were of school-age (5-17 years old).

Studies were not eligible if they met one of the following exclusion criteria:

- The study was no intervention study (e.g., theoretical paper, literature review, meta-analysis, or correlation study).

- Participants did not match the target population (e.g., university students or (pre-service) teachers).

- The study was published in a language other than English or German.

The selection of eligible studies was conducted in two stages. Firstly, we employed an initial screening of titles and abstracts against the inclusion and exclusion criteria. Screening procedures followed PRISMA guidelines (Moher et al., 2009). All studies were screened using the tool for systematic reviews Rayyan (Ouzzani et al., 2016). Rayyan is an open access online application that enables a semi-automated collaborative screening process. Secondly, all studies that appeared to meet the inclusion criteria, or when a decision could not be made based on the title and/or abstract, were screened again based on their full texts.

\subsection{Study Selection}

The described inclusion and exclusion criteria were applied during the selection process (for an overview of the study selection process see Figure 1). The initial search in the databases PsycINFO and PubPsych led to the identification of 521 records. Additionally, 13 records were identified by hand search. After removing duplicates, the titles, and abstracts of 479 records were screened for potential eligibility. This step led to the exclusion of 452 records. The full texts of 27 records were consequently assessed for eligibility. As a result, three more records were excluded. These steps led to the inclusion of 24 


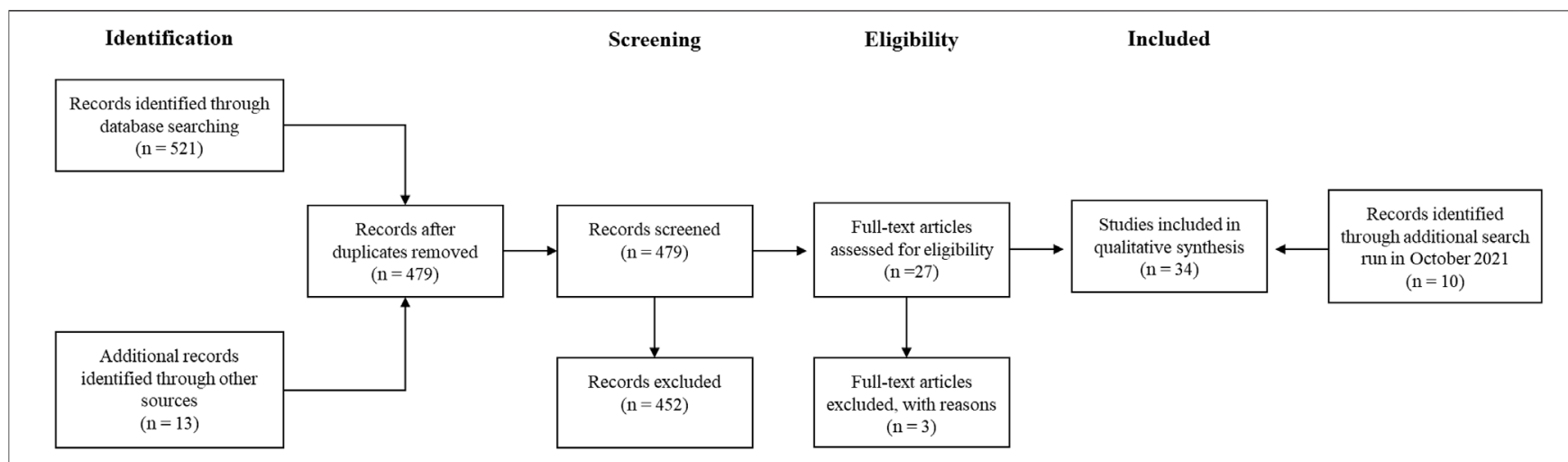

FIGURE 1 | Study selection process following PRISMA guidelines.

records. A second search run was conducted in October 2021 to include most recent studies. This led to the inclusion of ten more studies. The final number of studies for the qualitative synthesis was 34 .

\subsection{Data Extraction and Coding Procedure}

Next to general information about the studies, such as author(s), year of publication, and title, we extracted relevant data to address our specific research questions. Regarding our first research question (approaches, designs, and characteristics of existing interventions) we coded all information given by the author(s) about the study design, interventions, and their respective settings. This included information about the general study approach (quantitative, qualitative, mixed method), the study design (pre-post-test, follow up, control/ comparison group), the operationalization of $\mathrm{MA}$, as well as data about sample size and age group of the participants. Regarding the intervention we extracted information about the content as well as the intended goal of the interventions. We also coded the duration of the interventions (overall time span and number of sessions), the duration of single sessions, the intervention mode (computer-based, face-to-face), and the social arrangement (single, partner, small groups, class). Concerning our second research question (effects of these existing interventions) we coded the key results of the studies regarding the effectiveness of the intervention(s) to reduce MA as reported by the authors.

Relevant information has been coded using a spread sheet covering the previously described categories. The number of free text fields has been limited as much as possible to enable an unambiguous extraction and analysis of the data. Preferably fixed text such as yes/no decisions and drop-down lists has been used to code the data. The data extraction spread sheet has been previously piloted and adapted.

\section{RESULTS}

For a complete overview over all included studies (reference, sample, design, MA measure, operationalization type of MA, intervention, setting, and main findings) see Table $\mathbf{1}$.

\subsection{Intervention Approach}

Most of the included studies applied either a mathematical intervention (MI) approach (see section I in Table 1) or a cognitive-behavioral intervention (CBI) approach (see section II in Table 1). Four studies used other interventional approaches that could not clearly assigned to one of these two approaches (see section III in Table $\mathbf{1}$ ).

\subsection{Sample and Study Design}

The study samples differed between the two main intervention approaches (MI and $\mathrm{CBI}$ ) in regards to the age groups of the participants. $82 \%$ of the MI studies targeted school age children (6-12 years), whereas $57 \%$ of the studies within the CBI approach focused on adolescents (13-17 years). Regarding the sample size and choice of study design there appears to be no systematic difference between MI and CBI studies. The majority of the included studies applied a quantitative study design to examine the effects of various interventions on MA. Thereby, the sample size of the included studies varies strongly, $M=138(S D=171)$. Whilst some studies used large samples of over 300 participants (Shapka and Keating, 2003; Brandenberger and Moser, 2018; Vanbecelaere et al., 2020), other studies only collected information of approximately 20 participants (Kamann and Wong, 1993; Supekar et al., 2015; Choi-Koh and Ryoo, 2019). Most of the quantitative studies applied a pre-post design and included a control or comparison group. Whilst some studies used a waiting list procedure for the control group (i.e., the group received the same intervention with some time delay after the intervention group), other studies applied alternative interventions (e.g., Shapka and Keating, 2003; Asikhia and Mohangi, 2015) or applied modified version of the actual target intervention (Kramarski et al., 2010; Huang et al., 2014). Four studies additionally followed up on their participants in the intervention and control group (Sheffield and Hunt, 2006; Rauscher et al., 2017; Vanbecelaere et al., 2020; Vanbecelaere et al., 2021). Two of the identified studies applied single-case procedures to address potential effects of interventions on MA. LaGue et al. (2019) applied a multiple baseline approach within an experimental single-case design. Hord et al. (2018) used a qualitative approach to single-case research and focused on 
TABLE 1 | Overview of included studies.

\begin{tabular}{llll}
\hline Reference $\quad$ Sample & Mesign measure & $\begin{array}{c}\text { MA } \\
\text { operationali- } \\
\text { zation type }\end{array}$ & Intervention findings
\end{tabular}

\section{I - Mathematical Interventions (MI)}

\begin{tabular}{|c|c|c|c|c|c|c|c|}
\hline Alanazi (2020) & $\begin{array}{l}n=60 \\
\text { School age } \\
(6-12 \text { years })\end{array}$ & $\begin{array}{l}\text { quantitative; } \\
\text { pre-post; } \\
\text { control group }\end{array}$ & $\begin{array}{l}\text { Math Anxiety Scale } \\
\text { for children MASC; } \\
\text { Chiu and Henry } \\
\text { (1990) (Arabic } \\
\text { translation) }\end{array}$ & $\begin{array}{l}\text { Hyp/retro } \\
\text { (statelike-MA) } \\
\text { and anxiety } \\
\text { about failure in } \\
\text { math (trait-MA) }\end{array}$ & $\begin{array}{l}\text { Active recreational math } \\
\text { games (vs. regular math } \\
\text { teaching) }\end{array}$ & $\begin{array}{l}\text { Face-to-face; } \\
\text { Small group; } \\
8 \text { weeks; } 3 \\
\text { sessions } \\
\text { (45 min each) } \\
\text { per week }\end{array}$ & $\begin{array}{l}\text { Intervention group } \\
\text { obtained lower MA } \\
\text { scores and higher } \\
\text { math performance } \\
\text { than the control } \\
\text { group }\end{array}$ \\
\hline $\begin{array}{l}\text { Arias Rodriguez } \\
\text { et al. (2019) }\end{array}$ & $\begin{array}{l}n=42 \\
\text { School age } \\
(6-12 \text { years })\end{array}$ & $\begin{array}{l}\text { quantitative; } \\
\text { pre-post; } \\
\text { comparison } \\
\text { group }\end{array}$ & $\begin{array}{l}\text { Escala de } \\
\text { Ansiedade à } \\
\text { Matemática (Math } \\
\text { Anxiety Scale) EAM; } \\
\text { Carmo (2008) }\end{array}$ & $\begin{array}{l}\text { Hyp/retro } \\
\text { (statelike-MA) }\end{array}$ & $\begin{array}{l}\text { Numeracy musical training } \\
\text { Da Silva et al. (2017) (Low } \\
\text { vs. average achieving } \\
\text { students) }\end{array}$ & $\begin{array}{l}\text { Face-to-face; } \\
\text { Small group; } \\
8 \text { weeks; } 1 \\
\text { session } \\
\text { (40 min) per } \\
\text { week }\end{array}$ & $\begin{array}{l}\text { Both groups } \\
\text { obtained lower MA } \\
\text { scores and higher } \\
\text { math performance }\end{array}$ \\
\hline $\begin{array}{l}\text { Choi-Koh \& Ryoo, } \\
\text { (2019) }\end{array}$ & $\begin{array}{l}n=25 \\
\text { Adolescence } \\
\text { (13-17 years) }\end{array}$ & $\begin{array}{l}\text { quantitative; } \\
\text { pre-post; } \\
\text { comparison } \\
\text { group }\end{array}$ & $\begin{array}{l}\text { Math Anxiety Scale } \\
\text { for students (MASS) } \\
\text { revised by Ko and Yi } \\
\text { (2011) }\end{array}$ & $\begin{array}{l}\text { Hyp/retro } \\
\text { (statelike-MA) }\end{array}$ & $\begin{array}{l}\text { Quadratic functions } \\
\text { training and Brain } \\
\text { Integration in Education } \\
\text { program BIE; Kim (2010) } \\
\text { (Low MA vs. high MA) }\end{array}$ & $\begin{array}{l}\text { Face-to-face; } 3 \\
\text { lessons }\end{array}$ & $\begin{array}{l}\text { Reduction of MA in } \\
\text { high MA group; No } \\
\text { reduction of MA in } \\
\text { low MA group }\end{array}$ \\
\hline Hord et al. (2018) & $\begin{array}{l}n=2 \\
\text { Adolescence } \\
\text { (13-17 years) }\end{array}$ & $\begin{array}{l}\text { qualitative; } \\
\text { single case }\end{array}$ & $\begin{array}{l}\text { Field notes, } \\
\text { recordings, } \\
\text { interviews }\end{array}$ & Not classifiable & $\begin{array}{l}\text { Algebra training, individual } \\
\text { support }\end{array}$ & $\begin{array}{l}\text { Face-to-face; } \\
\text { Single }\end{array}$ & $\begin{array}{l}\text { One student needed } \\
\text { more support to } \\
\text { address MA than the } \\
\text { other; Both improved } \\
\text { math performance }\end{array}$ \\
\hline Huang et al. (2014) & $\begin{array}{l}n=56 \\
\text { School age } \\
(6-12 \text { years })\end{array}$ & $\begin{array}{l}\text { quantitative; } \\
\text { pre-post; } \\
\text { control group }\end{array}$ & $\begin{array}{l}\text { Math Anxiety Scale } \\
\text { for 1st and 2nd } \\
\text { grade students Shie } \\
\text { (2006) }\end{array}$ & $\begin{array}{l}\text { No further } \\
\text { information }\end{array}$ & $\begin{array}{l}\text { Digital game-based } \\
\text { learning with diagnostic } \\
\text { feedback (vs. without } \\
\text { diagnostic feedback) }\end{array}$ & $\begin{array}{l}\text { Computer; } \\
\text { Single; } \\
6 \text { weeks; } 2 \\
\text { sessions } \\
\text { (40 min each) } \\
\text { per week }\end{array}$ & $\begin{array}{l}\text { Both groups } \\
\text { obtained lower MA } \\
\text { scores and enhanced } \\
\text { levels of learning } \\
\text { motivation }\end{array}$ \\
\hline Jansen et al. (2013) & $\begin{array}{l}n=207 \\
\text { School age } \\
(6-12 \text { years })\end{array}$ & $\begin{array}{l}\text { quantitative; } \\
\text { pre-post; } \\
\text { control group }\end{array}$ & $\begin{array}{l}\text { Math Anxiety Scale } \\
\text { for children MASC; } \\
\text { Chiu and Henry } \\
\text { (1990) (Dutch } \\
\text { translation) }\end{array}$ & $\begin{array}{l}\text { Hyp/retro } \\
\text { (statelike-MA) } \\
\text { and anxiety } \\
\text { about failure in } \\
\text { math (trait-MA) }\end{array}$ & $\begin{array}{l}\text { Adaptive math training } \\
\text { software Math Garden } \\
\text { Klinkenberg et al. (2011) in } \\
\text { three experimental } \\
\text { conditions with different } \\
\text { pre-set success rates (vs. } \\
\text { regular math teaching) }\end{array}$ & $\begin{array}{l}\text { Computer; } \\
\text { Class; } \\
6 \text { weeks; } 4 \\
\text { sessions } \\
\text { (15 min each) } \\
\text { per week }\end{array}$ & $\begin{array}{l}\text { All groups obtained } \\
\text { lower MA scores; } \\
\text { Math performance } \\
\text { only improved in the } \\
\text { experimental } \\
\text { conditions }\end{array}$ \\
\hline $\begin{array}{l}\text { Kramarski et al. } \\
\text { (2010) }\end{array}$ & $\begin{array}{l}n=140 \\
\text { School age } \\
(6-12 \text { years })\end{array}$ & $\begin{array}{l}\text { quantitative; } \\
\text { pre-post; } \\
\text { control group }\end{array}$ & $\begin{array}{l}\text { Questionnaire } \\
\text { adapted from } \\
\text { Sarason (1980, } \\
\text { 1986) and Midgley } \\
\text { et al. (2000) }\end{array}$ & $\begin{array}{l}\text { Hyp/retro } \\
\text { (statelike-MA) } \\
\text { and anxiety } \\
\text { about failure in } \\
\text { math (trait-MA) }\end{array}$ & $\begin{array}{l}\text { Self-regulated math } \\
\text { learning (SRL) based on } \\
\text { the IMPROVE method } \\
\text { Mevarech and Kramarski } \\
\text { (1997) (vs. no SRL } \\
\text { support) }\end{array}$ & $\begin{array}{l}\text { Face-to-face; } \\
\text { Class; } \\
4 \text { weeks; } 4 \text { h } \\
\text { per week }\end{array}$ & $\begin{array}{l}\text { Intervention group } \\
\text { obtained lower MA } \\
\text { scores and higher } \\
\text { math problem solving } \\
\text { than the control } \\
\text { group }\end{array}$ \\
\hline $\begin{array}{l}\text { Lavasani et al. } \\
\text { (2012) }\end{array}$ & $\begin{array}{l}n=40 \\
\text { School age } \\
(6-12 \text { years })\end{array}$ & $\begin{array}{l}\text { quantitative; } \\
\text { pre-post; } \\
\text { control group }\end{array}$ & $\begin{array}{l}\text { Math Anxiety Scale } \\
\text { (18 items; no further } \\
\text { information) }\end{array}$ & $\begin{array}{l}\text { No further } \\
\text { information }\end{array}$ & $\begin{array}{l}\text { Cooperative learning (vs. } \\
\text { regular math teaching) }\end{array}$ & $\begin{array}{l}\text { Face-to-face; } \\
\text { Class; } 8 \\
\text { sessions }\end{array}$ & $\begin{array}{l}\text { Intervention group } \\
\text { obtained lower MA } \\
\text { scores and increased } \\
\text { help seeking behavior } \\
\text { than the control } \\
\text { group }\end{array}$ \\
\hline $\begin{array}{l}\text { Mehdizadeh et al. } \\
\text { (2013) }\end{array}$ & $\begin{array}{l}n=40 \\
\text { Adolescence } \\
\text { (13-17 years) }\end{array}$ & $\begin{array}{l}\text { quantitative; } \\
\text { pre-post; } \\
\text { control group }\end{array}$ & $\begin{array}{l}\text { Math Anxiety } \\
\text { Remote Sensing } \\
\text { Scale (MARS) by } \\
\text { Shokrani (2002) }\end{array}$ & $\begin{array}{l}\text { No further } \\
\text { information }\end{array}$ & $\begin{array}{l}\text { Cooperative learning (vs. } \\
\text { regular math teaching) }\end{array}$ & $\begin{array}{l}\text { Face-to-face; } \\
\text { Small group }\end{array}$ & $\begin{array}{l}\text { Intervention group } \\
\text { obtained lower MA } \\
\text { scores, higher math } \\
\text { performance, and } \\
\text { increased help } \\
\text { seeking behavior } \\
\text { than the control } \\
\text { group }\end{array}$ \\
\hline $\begin{array}{l}\text { Mevarech et al. } \\
\text { (1991) }\end{array}$ & $\begin{array}{l}n=149 \\
\text { School age } \\
(6-12 \text { years })\end{array}$ & $\begin{array}{l}\text { quantitative; } \\
\text { pre-post; } \\
\text { comparison } \\
\text { group }\end{array}$ & $\begin{array}{l}\text { Math Anxiety Scale } \\
\text { by Mevarech and } \\
\text { Rich (1985) }\end{array}$ & $\begin{array}{l}\text { Hyp/retro } \\
\text { (statelike-MA) } \\
\text { and anxiety } \\
\text { about failure in } \\
\text { math (trait-MA) }\end{array}$ & $\begin{array}{l}\text { Computer assisted math } \\
\text { instruction TOAM system; } \\
\text { Hativa et al. (1990) } \\
\text { (Cooperative use in pairs } \\
\text { vs. individual use) }\end{array}$ & $\begin{array}{l}\text { Computer; } \\
\text { Pairs; } 20 \text { min } \\
\text { per session }\end{array}$ & $\begin{array}{l}\text { Low achieving } \\
\text { students in } \\
\text { cooperative group } \\
\text { obtained lower MA } \\
\text { scores and higher } \\
\text { math performance } \\
\text { d on following page) }\end{array}$ \\
\hline
\end{tabular}


TABLE 1 | (Continued) Overview of included studies.

\begin{tabular}{|c|c|c|c|c|c|c|c|}
\hline Reference & Sample & Design & MA measure & $\begin{array}{l}\text { MA } \\
\text { operationali- } \\
\text { zation type }^{a}\end{array}$ & Intervention & Setting & Main findings \\
\hline $\begin{array}{l}\text { Passolunghi et al. } \\
\text { (2020) }\end{array}$ & $\begin{array}{l}n=224 \\
\text { School age } \\
(6-12 \text { years })\end{array}$ & $\begin{array}{l}\text { quantitative; } \\
\text { pre-post; } \\
\text { control group }\end{array}$ & $\begin{array}{l}\text { Abbreviated Math } \\
\text { Anxiety Scale } \\
\text { (AMAS; Caviola } \\
\text { et al., 2017b) }\end{array}$ & $\begin{array}{l}\text { Hyp/retro } \\
\text { (statelike-MA) }\end{array}$ & $\begin{array}{l}\text { Calculation strategies } \\
\text { training (vs. control } \\
\text { training) }\end{array}$ & $\begin{array}{l}\text { Face-to-face; } \\
\text { Small group; } \\
8 \text { weeks; } 1 \\
\text { session ( } 60 \text { min } \\
\text { each) per week }\end{array}$ & $\begin{array}{l}\text { than in individual } \\
\text { group; Both groups } \\
\text { showed similar math } \\
\text { self-concept } \\
\text { Intervention group } \\
\text { obtained lower MA } \\
\text { scores and higher } \\
\text { math performance } \\
\text { than the control } \\
\text { group }\end{array}$ \\
\hline $\begin{array}{l}\text { Rauscher et al. } \\
\text { (2017) }\end{array}$ & $\begin{array}{l}n=68 \\
\text { School age } \\
(6-12 \text { years })\end{array}$ & $\begin{array}{l}\text { quantitative; } \\
\text { pre-post-follow } \\
\text { up; control } \\
\text { group }\end{array}$ & $\begin{array}{l}\text { MAl Kohn et al. } \\
\text { (2013) }\end{array}$ & $\begin{array}{l}\text { Hyp/retro } \\
\text { (statelike-MA) }\end{array}$ & $\begin{array}{l}\text { Adaptive math training } \\
\text { Calcularis e.g., Käser et al. } \\
\text { (2013) (vs. waiting list vs. } \\
\text { control training) }\end{array}$ & $\begin{array}{l}\text { Computer; } \\
\text { Single; } \\
6 \text { weeks; } 5 \\
\text { sessions } \\
\text { (20 min each) } \\
\text { per week }\end{array}$ & $\begin{array}{l}\text { Intervention group } \\
\text { obtained lower MA } \\
\text { scores than waiting } \\
\text { list control group; No } \\
\text { difference in MA } \\
\text { between intervention } \\
\text { group and control } \\
\text { training group; All } \\
\text { groups improved } \\
\text { similarly in their } \\
\text { attitude towards } \\
\text { math and math self- } \\
\text { concept }\end{array}$ \\
\hline $\begin{array}{l}\text { Supekar et al. } \\
\text { (2015) }\end{array}$ & $\begin{array}{l}n=28 \\
\text { School age } \\
(6-12 \text { years })\end{array}$ & $\begin{array}{l}\text { quantitative; } \\
\text { pre-post; } \\
\text { comparison } \\
\text { group }\end{array}$ & $\begin{array}{l}\text { Math Anxiety Level } \\
\text { SEMA Wu et al. } \\
(2012)\end{array}$ & $\begin{array}{l}\text { Hyp/retro } \\
\text { (statelike-MA) }\end{array}$ & $\begin{array}{l}\text { Adaptation of MathWise } \\
\text { Fuchs et al. (2013) (Low vs. } \\
\text { high MA) }\end{array}$ & $\begin{array}{l}\text { Face-to-face; } \\
8 \text { weeks; } \\
\text { Single; } 3 \\
\text { sessions } \\
\text { (45 min each) } \\
\text { per week }\end{array}$ & $\begin{array}{l}\text { Reduction of MA in } \\
\text { High MA group; Math } \\
\text { performance } \\
\text { improved equally in } \\
\text { both groups }\end{array}$ \\
\hline Tok (2013) & $\begin{array}{l}n=55 \\
\text { School age } \\
(6-12 \text { years })\end{array}$ & $\begin{array}{l}\text { quantitative; } \\
\text { pre-post; } \\
\text { control group }\end{array}$ & $\begin{array}{l}\text { Math Anxiety Scale } \\
\text { by Bindak (2005) }\end{array}$ & $\begin{array}{l}\text { Hyp/retro } \\
\text { (statelike-MA) } \\
\text { and anxiety } \\
\text { about failure in } \\
\text { math (trait-MA) }\end{array}$ & $\begin{array}{l}\text { Metacognitive strategy } \\
\text { training (Know-Want- } \\
\text { Learn-Strategy) (vs. } \\
\text { regular math teaching) }\end{array}$ & $\begin{array}{l}\text { Face-to-face; } \\
\text { Class; } \\
8 \text { weeks; } 4 \\
\text { sessions } \\
\text { (40 min each) } \\
\text { per week }\end{array}$ & $\begin{array}{l}\text { Intervention group } \\
\text { showed no reduction } \\
\text { of MA but obtained } \\
\text { higher math } \\
\text { performance and } \\
\text { metacognition than } \\
\text { the control group }\end{array}$ \\
\hline Tok et al. (2015) & $\begin{array}{l}n=42 \\
\text { School age } \\
(6-12 \text { years })\end{array}$ & $\begin{array}{l}\text { quantitative; } \\
\text { pre-post; } \\
\text { control group }\end{array}$ & $\begin{array}{l}\text { Math Anxiety Scale } \\
\text { by Bindak (2005) }\end{array}$ & $\begin{array}{l}\text { Hyp/retro } \\
\text { (statelike-MA) } \\
\text { and anxiety } \\
\text { about failure in } \\
\text { math (trait-MA) }\end{array}$ & $\begin{array}{l}\text { Teaching math creatively } \\
\text { (vs. regular math teaching) }\end{array}$ & $\begin{array}{l}\text { Face-to-face; } \\
\text { Class; } \\
6 \text { weeks; } 4 \\
\text { sessions } \\
\text { (40 min each) } \\
\text { per week }\end{array}$ & $\begin{array}{l}\text { Intervention group } \\
\text { obtained lower MA } \\
\text { scores, higher math } \\
\text { performance, and } \\
\text { better attitudes } \\
\text { towards math than } \\
\text { the control group }\end{array}$ \\
\hline $\begin{array}{l}\text { Vanbecelaere et al. } \\
(2020)\end{array}$ & $\begin{array}{l}n=336 \\
\text { School age } \\
(6-12 \text { years })\end{array}$ & $\begin{array}{l}\text { quantitative; } \\
\text { pre-post-follow } \\
\text { up; control } \\
\text { group }\end{array}$ & $\begin{array}{l}\text { Child Math Anxiety } \\
\text { Questionnaire - } \\
\text { Revised CMAQ-R; } \\
\text { Ramirez et al. (2016) } \\
\text { (Flemish adaptation) }\end{array}$ & $\begin{array}{l}\text { Hyp/retro } \\
\text { (statelike-MA) }\end{array}$ & $\begin{array}{l}\text { Digital game-based } \\
\text { learning in reading and } \\
\text { math (vs. regular math } \\
\text { teaching) }\end{array}$ & $\begin{array}{l}\text { Computer; } \\
8 \text { weeks; } \\
\text { Single; 1-2 } \\
\text { sessions } \\
\text { (50 min each) } \\
\text { per week }\end{array}$ & $\begin{array}{l}\text { Intervention group } \\
\text { showed no reduction } \\
\text { of MA but performed } \\
\text { better in number line } \\
\text { estimation and } \\
\text { reading competence } \\
\text { than the control } \\
\text { group }\end{array}$ \\
\hline $\begin{array}{l}\text { Vanbecelaere et al. } \\
\text { (2021) }\end{array}$ & $\begin{array}{l}n=78 \\
\text { School age } \\
(6-12 \text { years })\end{array}$ & $\begin{array}{l}\text { quantitative; } \\
\text { pre-post-follow } \\
\text { up; } \\
\text { comparison } \\
\text { group }\end{array}$ & $\begin{array}{l}\text { State-Math Anxiety } \\
\text { Questionnaire state- } \\
\text { MAQ; e.g., Orbach } \\
\text { et al. (2020) (Dutch } \\
\text { translation) }\end{array}$ & $\begin{array}{l}\text { State-MA (real- } \\
\text { time assessment) }\end{array}$ & $\begin{array}{l}\text { Adaptive educational math } \\
\text { game Number Sense } \\
\text { Game, Maertens et al. } \\
\text { (2016) (vs. non-adaptive } \\
\text { version) }\end{array}$ & $\begin{array}{l}\text { Computer; } \\
3 \text { weeks; } \\
\text { Single; } 2 \\
\text { sessions } \\
\text { (30 min each) } \\
\text { per week }\end{array}$ & $\begin{array}{l}\text { Both groups } \\
\text { obtained lower MA } \\
\text { scores and improved } \\
\text { on early numeracy } \\
\text { skills }\end{array}$ \\
\hline
\end{tabular}

\section{II - Cognitive-behavioral Interventions (CBI)}

\begin{tabular}{lllll}
\hline Asanjarani and & $n=30$ & quantitative; & Math Anxiety Rating & Hyp/retro \\
Zarebahramabadi & School age & pre-post; & Scale (MARS) by & (statelike-MA) \\
(2021) & (6-12 years) & control group & &
\end{tabular}

\begin{tabular}{|c|c|c|}
\hline $\begin{array}{l}\text { Cognitive behavioral } \\
\text { therapy based on Chiu and } \\
\text { Henry (1990) (vs. control }\end{array}$ & $\begin{array}{l}\text { Face-to-face; } \\
\text { Small group; } 1\end{array}$ & $\begin{array}{l}\text { Intervention group } \\
\text { obtained lower MA } \\
\text { scores and higher }\end{array}$ \\
\hline
\end{tabular}


TABLE 1 | (Continued) Overview of included studies.

\begin{tabular}{|c|c|c|c|c|c|c|c|}
\hline Reference & Sample & Design & MA measure & $\begin{array}{l}\text { MA } \\
\text { operationali- } \\
\text { zation type }^{a}\end{array}$ & Intervention & Setting & Main findings \\
\hline & & & $\begin{array}{l}\text { Suinn and Winston } \\
\text { (2003) }\end{array}$ & & $\begin{array}{l}\text { group not further } \\
\text { described) }\end{array}$ & $\begin{array}{l}\text { session (90 min } \\
\text { each) per week }\end{array}$ & $\begin{array}{l}\text { math self-concept } \\
\text { than the control } \\
\text { group }\end{array}$ \\
\hline $\begin{array}{l}\text { Asikhia and } \\
\text { Mohangi (2015) }\end{array}$ & $\begin{array}{l}n=120 \\
\text { Adolescence } \\
(13-17 \text { years })\end{array}$ & $\begin{array}{l}\text { quantitative; } \\
\text { pre-post; } \\
\text { control group }\end{array}$ & $\begin{array}{l}\text { Math Anxiety Rating } \\
\text { Scale - Revised } \\
\text { MARS-R, Plake and } \\
\text { Parker (1982) }\end{array}$ & $\begin{array}{l}\text { Hyp/retro } \\
\text { (statelike-MA) }\end{array}$ & $\begin{array}{l}\text { Problem solving training } \\
\text { (vs. class debate) }\end{array}$ & $\begin{array}{l}\text { Face-to-face; } 8 \\
\text { sessions } \\
\text { (60 min each) }\end{array}$ & $\begin{array}{l}\text { Intervention group } \\
\text { obtained lower MA } \\
\text { scores than the } \\
\text { control group }\end{array}$ \\
\hline $\begin{array}{l}\text { Brandenberger and } \\
\text { Moser (2018) }\end{array}$ & $\begin{array}{l}n=348 \\
\text { Adolescence } \\
(13-17 \text { years })\end{array}$ & $\begin{array}{l}\text { quantitative; } \\
\text { pre-post; } \\
\text { control group }\end{array}$ & $\begin{array}{l}\text { Achievement } \\
\text { Emotions } \\
\text { Questionnaire - } \\
\text { Mathematics (AEQ- } \\
\text { M) - German Pekrun } \\
\text { et al. (2005) } \\
\text { (Shortened version) }\end{array}$ & $\begin{array}{l}\text { Hyp/retro } \\
\text { (statelike-MA) }\end{array}$ & $\begin{array}{l}\text { Combined student and } \\
\text { teacher workshops on } \\
\text { e.g., emotions, motivation, } \\
\text { learning goals, cooperative } \\
\text { learning, and feedback (vs. } \\
\text { student workshops only } \\
\text { vs. waiting list) }\end{array}$ & $\begin{array}{l}\text { Face-to-face; } \\
38 \text { weeks; } \\
\text { Class; 3-4 } \\
\text { sessions } \\
\text { (45 min each) }\end{array}$ & $\begin{array}{l}\text { All groups obtained } \\
\text { lower MA scores; } \\
\text { Intervention group } \\
\text { reported higher joy of } \\
\text { learning than the } \\
\text { waiting list control } \\
\text { group and the } \\
\text { student workshops } \\
\text { only group }\end{array}$ \\
\hline $\begin{array}{l}\text { Collingwood and } \\
\text { Dewey (2018) }\end{array}$ & $\begin{array}{l}n=144 \\
\text { School age } \\
(6-12 \text { years })\end{array}$ & $\begin{array}{l}\text { quantitative; } \\
\text { pre-post; } \\
\text { control group }\end{array}$ & $\begin{array}{l}\text { Scale of Math } \\
\text { Anxiety Cavanaugh } \\
\text { and Sparrow (2011); } \\
\text { Math Anxiety Scale } \\
\text { OECD (2005) }\end{array}$ & $\begin{array}{l}\text { Hyp/retro } \\
\text { (statelike-MA) }\end{array}$ & $\begin{array}{l}\text { Coping strategies; mindful } \\
\text { breathing; self-regulation } \\
\text { (vs. waiting list) }\end{array}$ & $\begin{array}{l}\text { Face-to-face; } \\
\text { Small group; } \\
4 \text { weeks; } 3 \\
\text { sessions } \\
\text { (45 min each) } \\
\text { per week }\end{array}$ & $\begin{array}{l}\text { Intervention group } \\
\text { showed no reduction } \\
\text { of MA or } \\
\text { enhancement of } \\
\text { math self-concept } \\
\text { but higher math } \\
\text { performance than the } \\
\text { control group }\end{array}$ \\
\hline Hines et al. (2016) & $\begin{array}{l}n=93 \\
\text { Adolescence } \\
(13-17 \text { years })\end{array}$ & $\begin{array}{l}\text { quantitative; } \\
\text { pre-post; } \\
\text { control group }\end{array}$ & $\begin{array}{l}\text { Math Anxiety Rating } \\
\text { Scale MARS, Suinn } \\
\text { and Edwards (1982) }\end{array}$ & $\begin{array}{l}\text { Hyp/retro } \\
\text { (statelike-MA) }\end{array}$ & $\begin{array}{l}\text { Expressive writing on } \\
\text { feelings about math (vs. } \\
\text { expressive writing on } \\
\text { neutral topic) }\end{array}$ & $\begin{array}{l}\text { Single; } \\
\text { 15-30 min a } \\
\text { day for } 3 \text { days }\end{array}$ & $\begin{array}{l}\text { Intervention group } \\
\text { reported reduced } \\
\text { levels of general and } \\
\text { MA and the control } \\
\text { group had a } \\
\text { reduction in MA. }\end{array}$ \\
\hline $\begin{array}{l}\text { Kamann and Wong } \\
\text { (1993) }\end{array}$ & $\begin{array}{l}n=20 \\
\text { School age } \\
(6-12 \text { years })\end{array}$ & $\begin{array}{l}\text { quantitative; } \\
\text { pre-post; } \\
\text { comparison } \\
\text { group }\end{array}$ & $\begin{array}{l}\text { Think out loud (self- } \\
\text { talk measure) }\end{array}$ & Not classifiable & $\begin{array}{l}\text { Coping strategy training } \\
\text { (students with learning } \\
\text { disability (LD) vs. students } \\
\text { without LD) }\end{array}$ & $\begin{array}{l}\text { Face-to-face; } \\
\text { Small group; } \\
6 \text { weeks; } 1 \\
\text { session per } \\
\text { week }\end{array}$ & $\begin{array}{l}\text { LD group showed } \\
\text { increase in positive } \\
\text { self-talk compared to } \\
\text { group without LD } \\
\text { group indicating } \\
\text { enhanced coping } \\
\text { with MA. }\end{array}$ \\
\hline $\begin{array}{l}\text { Karimi and } \\
\text { Venkatesan (2009) }\end{array}$ & $\begin{array}{l}n=33 \\
\text { Adolescence } \\
(13-17 \text { years })\end{array}$ & $\begin{array}{l}\text { quantitative; } \\
\text { pre-post; } \\
\text { control group }\end{array}$ & $\begin{array}{l}\text { Math Anxiety Rating } \\
\text { Scale MARS, } \\
\text { Alexander and } \\
\text { Martray (1989) }\end{array}$ & $\begin{array}{l}\text { Hyp/retro } \\
\text { (statelike-MA) }\end{array}$ & $\begin{array}{l}\text { Cognitive behavior group } \\
\text { therapy (vs. control group } \\
\text { not further described) }\end{array}$ & $\begin{array}{l}\text { Face-to-face; } \\
\text { Small group; } \\
7.5 \text { weeks; } 2 \\
\text { sessions } \\
\text { (90 min each) } \\
\text { per week }\end{array}$ & $\begin{array}{l}\text { Intervention group } \\
\text { obtained lower MA } \\
\text { scores than the } \\
\text { control group }\end{array}$ \\
\hline Kim et al. (2017) & $\begin{array}{l}n=138 \\
\text { Adolescence } \\
(13-17 \text { years })\end{array}$ & $\begin{array}{l}\text { quantitative; } \\
\text { pre-post; } \\
\text { control group }\end{array}$ & $\begin{array}{l}\text { Math Anxiety Rating } \\
\text { Scale - Revised } \\
\text { MARS-R, Plake and } \\
\text { Parker (1982) }\end{array}$ & $\begin{array}{l}\text { Hyp/retro } \\
\text { (statelike-MA) }\end{array}$ & $\begin{array}{l}\text { Embodied agent with } \\
\text { instructional guidance and } \\
\text { anxiety treating messages } \\
\text { (vs. embodied agent with } \\
\text { instructional guidance } \\
\text { only) }\end{array}$ & $\begin{array}{l}\text { Computer; } \\
\text { Class; } 1 \text { week; } \\
4 \text { sessions } \\
\text { (45 min each) } \\
\text { per week }\end{array}$ & $\begin{array}{l}\text { Both groups } \\
\text { obtained lower MA } \\
\text { scores and higher } \\
\text { math performance }\end{array}$ \\
\hline LaGue et al. (2019) & $\begin{array}{l}n=3 \\
\text { Adolescence } \\
(13-17 \text { years })\end{array}$ & $\begin{array}{l}\text { quantitative; } \\
\text { single case } \\
\text { design with } \\
\text { multiple } \\
\text { baselines }\end{array}$ & $\begin{array}{l}\text { Fennema-Sherman } \\
\text { Math Anxiety Scale - } \\
\text { Revised (FSMAS-R: } \\
\text { FSANX subscale) }\end{array}$ & $\begin{array}{l}\text { Hyp/retro } \\
\text { (statelike-MA) }\end{array}$ & $\begin{array}{l}\text { Mindfulness-based } \\
\text { cognitive therapy }\end{array}$ & $\begin{array}{l}\text { Face-to-face; } \\
\text { Single; } \\
6 \text { weeks; } 2 \\
\text { sessions } \\
\text { ( } 45 \text { min each) } \\
\text { per week }\end{array}$ & $\begin{array}{l}\text { All three students } \\
\text { showed decreased } \\
\text { levels of MA. }\end{array}$ \\
\hline $\begin{array}{l}\text { Passolunghi et al. } \\
\text { (2020) }\end{array}$ & $\begin{array}{l}n=224 \\
\text { School age } \\
(6-12 \text { years })\end{array}$ & $\begin{array}{l}\text { quantitative; } \\
\text { pre-post; } \\
\text { control group }\end{array}$ & $\begin{array}{l}\text { Abbreviated Math } \\
\text { Anxiety Scale } \\
\text { (AMAS; Caviola } \\
\text { et al., 2017b) }\end{array}$ & $\begin{array}{l}\text { Hyp/retro } \\
\text { (statelike-MA) }\end{array}$ & $\begin{array}{l}\text { Identifying and coping with } \\
\text { MA related feelings (vs. } \\
\text { control training) }\end{array}$ & $\begin{array}{l}\text { Face-to-face; } \\
\text { Small group; } \\
8 \text { weeks; } 1 \\
\text { session ( } 60 \text { min } \\
\text { each) per week }\end{array}$ & $\begin{array}{l}\text { Intervention group } \\
\text { obtained lower MA } \\
\text { scores but no } \\
\text { increase in math } \\
\text { performance } \\
\text { compared to the } \\
\text { control group } \\
\text { d on following page) }\end{array}$ \\
\hline
\end{tabular}


TABLE 1 | (Continued) Overview of included studies.

\begin{tabular}{|c|c|c|c|c|c|c|c|}
\hline Reference & Sample & Design & MA measure & $\begin{array}{l}\text { MA } \\
\text { operationali- } \\
\text { zation type }^{a}\end{array}$ & Intervention & Setting & Main findings \\
\hline Ruark (2021) & $\begin{array}{l}n=40 \\
\text { Adolescence } \\
\text { (13-17 years) }\end{array}$ & $\begin{array}{l}\text { quantitative; } \\
\text { pre-post; } \\
\text { control group }\end{array}$ & $\begin{array}{l}\text { Modified } \\
\text { Abbreviated Math } \\
\text { Anxiety Scale } \\
\text { (mAMAS; Carey } \\
\text { et al., 2017) }\end{array}$ & $\begin{array}{l}\text { Hyp/retro } \\
\text { (statelike-MA) }\end{array}$ & $\begin{array}{l}\text { Expressive writing on math } \\
\text { homework problems and } \\
\text { feelings (vs. expressive } \\
\text { writing on homework } \\
\text { problems only) }\end{array}$ & $\begin{array}{l}\text { Single; } \\
2 \text { weeks; } \\
\text { every day }\end{array}$ & $\begin{array}{l}\text { No reduction of MA in } \\
\text { both groups }\end{array}$ \\
\hline $\begin{array}{l}\text { Ruff and Boes } \\
\text { (2014) }\end{array}$ & $\begin{array}{l}n=13 \\
\text { School age } \\
(6-12 \text { years })\end{array}$ & $\begin{array}{l}\text { Mixed-method; } \\
\text { pre-post }\end{array}$ & $\begin{array}{l}\text { Math Anxiety Scale } \\
\text { for children MASC; } \\
\text { Chiu and Henry } \\
\text { (1990); Five open } \\
\text { ended (self- } \\
\text { developed) } \\
\text { questions }\end{array}$ & $\begin{array}{l}\text { Hyp/retro } \\
\text { (statelike-MA) } \\
\text { and anxiety } \\
\text { about failure in } \\
\text { math (trait-MA) }\end{array}$ & $\begin{array}{l}\text { School counseling (e.g., } \\
\text { identifying and expressing } \\
\text { feelings; stress reduction, } \\
\text { and relaxation) }\end{array}$ & $\begin{array}{l}\text { Face-to-face; } \\
\text { Small group; } \\
6 \text { weeks; } 2 \\
\text { sessions per } \\
\text { weeks }\end{array}$ & $\begin{array}{l}\text { Some students } \\
\text { obtained lower MA } \\
\text { scores and higher } \\
\text { math performance } \\
\text { compared to the pre- } \\
\text { test; Teachers } \\
\text { reported more } \\
\text { confidence and } \\
\text { participation in math } \\
\text { class }\end{array}$ \\
\hline $\begin{array}{l}\text { Sheffield and Hunt } \\
\text { (2006) }\end{array}$ & $\begin{array}{l}n=154 \\
\text { School age } \\
(6-12 \text { years })\end{array}$ & $\begin{array}{l}\text { quantitative; } \\
\text { pre-post-follow } \\
\text { up; control } \\
\text { group }\end{array}$ & $\begin{array}{l}\text { Maths Anxiety } \\
\text { Rating Scale for } \\
\text { Children MASC, } \\
\text { Chiu and Henry } \\
\text { (1990) }\end{array}$ & $\begin{array}{l}\text { Hyp/retro } \\
\text { (statelike-MA) } \\
\text { and anxiety } \\
\text { about failure in } \\
\text { math (trait-MA) }\end{array}$ & $\begin{array}{l}\text { Systematic desensitization } \\
\text { modified from } \\
\text { Meichenbaum (1977) (vs. } \\
\text { classroom games) }\end{array}$ & $\begin{array}{l}\text { Face-to-face; } 1 \\
\text { session; } 1 \mathrm{~h}\end{array}$ & $\begin{array}{l}\text { Intervention group } \\
\text { obtained lower MA } \\
\text { scores and higher } \\
\text { math performance } \\
\text { than the control } \\
\text { group }\end{array}$ \\
\hline Singh (2016) & $\begin{array}{l}n=60 \\
\text { Adolescence } \\
\text { (13-17 years) }\end{array}$ & $\begin{array}{l}\text { quantitative; } \\
\text { pre-post }\end{array}$ & $\begin{array}{l}\text { Short Math Anxiety } \\
\text { Rating Scale } \\
\text { (sMARS) based on } \\
\text { MARS Richardson } \\
\text { and Suinn (1972) }\end{array}$ & $\begin{array}{l}\text { Hyp/retro } \\
\text { (statelike-MA) }\end{array}$ & $\begin{array}{l}\text { Behavior modification; } \\
\text { Super brain yoga }\end{array}$ & $\begin{array}{l}\text { Face-to-face; } \\
6 \text { weeks }\end{array}$ & $\begin{array}{l}\text { Students obtained } \\
\text { lower MA scores and } \\
\text { higher math } \\
\text { performance } \\
\text { compared to the pre- } \\
\text { test }\end{array}$ \\
\hline
\end{tabular}

\section{III - Other Interventions}

Idris (2006)

$n=109$

Adolescence

(13-17 years)

quantitative;

pre-post;

control group

Math Anxiety Scale

(no further

information)

Segumpan and Tan $n=90$

(2018)

Adolescence

(13-17 years)

quantitative;

Math Self-Efficacy

pre-post-follow and Anxiety

up; control

Questionnaire

(MSEAQ; May,

2009) (Adapted

version)

Shapka and

$n=786$

Keating (2003)

Adolescence

(13-17 years)

quantitative;

pre-post;

control group

perceived Math

Anxiety and attitude

towards math

No further

information
Hyp/retro

(statelike-MA)

and anxiety

about failure in

math (trait-MA)

Hyp/retro
(statelike-MA)
Graphing calculator (vs. no graphing calculator)

Fa

Class;

10 weeks

Flipped classroom (vs.

regular math teaching)

Computer and

face-to-face;

Single and

class

Girls-only math teaching

(vs. mixed gender

teaching)

Face-to-face;

Class
Intervention group obtained lower MA scores and higher math performance scores than the control group Intervention group obtained lower MA scores than the control group; Both groups increased math performance No reduction of MA or increase in perceived math competence in intervention group (single sex girls) but higher math performance and course enrolment than in control group (co-ed girls)

$\begin{array}{lll}\text { Hyp/retro } & \text { Neuropsychological } & \text { Computer; } \\ \text { (statelike-MA) } & \text { feedback while playing } & \text { Single; } 2 \\ & \text { math computer game } & \text { sessions in } \\ & \text { (Math-Mind game; } & 2 \text { days; } 4 \text { data } \\ \text { developed for this study) } & \text { gathering } \\ & \text { waves per } \\ & \text { session }\end{array}$

\footnotetext{
Fennema-Sherman

Math Anxiety Scale
FSMAS, Kazelskis
and Reeves (2002)

Math Anxiety Scale
FSMAS, Kazelskis
and Reeves (2002)

Math Anxiety Scale
FSMAS, Kazelskis
and Reeves (2002)

Adolescence

quantitative

(13-17 years)

Wet (2015)
} 
two eighth grade students with learning disabilities using a descriptive, qualitative microanalysis.

\subsection{MA Measure}

Different quantitative measures have been used to assess the level of MA (for an overview see Table 1). Some of the measures have been extensively researched and validated, such as the Math Anxiety Scale for children (MASC; Chiu and Henry, 1990) or the Math Anxiety Rating Scale-Revised (MARS-R; Plake and Parker, 1982). Often measures were translated and/or adapted for the specific contexts and needs of the studies. Some studies used measures that were self-developed or not as commonly known (e.g., Kramarski et al., 2010; Tok et al., 2015; Singh, 2016) Also, qualitative measures such as observational field notes and selftalk recordings have been used (Kamann and Wong, 1993; Hord et al., 2018). According to the differentiations by Sorvo et al. (2017) and Orbach et al. (2019), one study (Vanbecelaere et al., 2021) used a real-time assessment measuring individuals mathrelated anxiety reaction during a math test situation (state-MA), 19 studies (approx. 54\%) applied questionnaires with hypothetical/retrospective questions asking how anxious the individual would feel during a math-related situation (anxiety in math-related situations/statelike-MA) and nine studies (approx. 26\%) used questionnaires with hypothetical/ retrospective questions about anxiety in math-related situations (statelike-MA) and questions focusing anxiety about failure in math (trait-MA). Two studies used unclassifiable qualitative approaches (Kamann and Wong, 1993; Hord et al., 2018). Four studies provided no clear information about the MA operationalization (Idris, 2006; Lavasani et al., 2012; Mehdizadeh et al., 2013; Huang et al., 2014).

\subsection{Intervention Activity}

\subsubsection{Mathematical Interventions}

The MI covered a wide range of different activities and programs, such as educational games or formalized math programs. Due to the amount of activities, only selected studies are presented in more detail below. The study selection does not constitute an evaluation of the quality of the studies. For a comprehensive overview of all MI see the first section of Table 1.

Alanazi (2020), Huang et al. (2014), and Vanbecelaere et al. (2021) investigated the effect of educational math games on MA and performance in primary school children. The intervention group in Alanazi (2020) study participated in face-to-face recreational math games (e.g., movement games containing mathematical problems) in addition to their regular math teaching. The comparison group received regular math teaching. The intervention group obtained lower MA scores and higher math performance than the control group. Huang et al. (2014) and Vanbecelaere et al. (2021) applied a digital game-based learning approach. Huang et al. (2014) designed a digital math game to train basic arithmetic operations that provided the children in the intervention group with interactive diagnostic feedback. The children in the comparison group also played the game but without diagnostic feedback. Both groups obtained lower MA scores and enhanced levels of learning motivation. Vanbecelaere et al. (2021) compared an adaptive version with a nonadaptive version of the Number Sense Game (Maertens et al., 2016). The Number Sense Game contained two types of exercises, a comparison game and a number line estimation game. Both groups obtained lower MA scores and improved on early numeracy skills.

Jansen et al. (2013), Rauscher et al. (2017), and Supekar et al. (2015) investigated the effect of formalized math training programs on primary school students' math performance and anxiety. Jansen et al. (2013) and Rauscher et al. (2017) applied specific math training software, namely Math Garden (Klinkenberg et al., 2011) and Calcularis (Käser et al., 2013). In Jansen et al. (2013) study the control group received regular math teaching. Both groups obtained lower MA scores and the math performance only improved in the intervention group. Rauscher et al. (2017) compared the intervention group with two control groups; one was a waiting list group, the other received a control training. The results showed that the intervention group obtained lower MA scores than the waiting list control group, but there was no difference in MA between the intervention group and the control training group. Supekar et al. (2015) examined an adaption of MathWise (Fuchs et al., 2013), a training program that aims to improve number knowledge, counting speed and the application of calculation strategies. Comparing children with high MA and low MA levels, the children with high MA significantly decreased their MA. In regards to math performance both groups benefited equally from the training.

\subsubsection{Cognitive-Behavioral Interventions}

The CBI also included different techniques and activities, such as coping strategy training or expressive writing. Due to the amount of activities, only selected studies are presented in more detail below. The study selection does not constitute an evaluation of the quality of the studies. For a comprehensive overview of all CBI see the second section of Table 1 .

Collingwood and Dewey (2018), Kamann and Wong (1993), Passolunghi et al. (2020), and Ruff and Boess (2014) investigated the effect of coping strategy trainings on primary school students' MA. Kamann and Wong (1993) examined a coping strategy based on cognitive behavior modification (Meichenbaum, 1977) to reduce MA. They compared children with and without learning disabilities (LD) providing both groups with sample self-instruction statements on cue cards to assist them in applying those statements at each level of the coping process. The LD group showed increased positive self-talk compared to the group without LD indicating enhanced coping with MA. Collingwood and Dewey (2018) examined a multi-dimensional cognitive intervention called Thinking your problems away (Martin, 2008) that encouraged (among other things such as selfregulation) the use of positive-self-coping statements based on Kamann and Wong (1993). The control group was a waiting list control group. The intervention group showed no reduction of MA or enhancement of math self-concept but higher math performance than the control group. Passolunghi et al. (2020) trained the primary school children in strategy-based techniques (among others things such as the recognition of emotions) to decrease their MA. These techniques included breathing 
exercises, safe place visualizations and re-appraisal of negative thoughts based on Ellis and Bernard (2006). The control group received a control training composed of playful activities with comic strips. The intervention group obtained lower MA scores but no increase in math performance compared to the control group.

Hines et al. (2016) and Ruark (2021) investigated the effect of expressive writing on MA in secondary school students. In the intervention group of Hines et al. (2016) study the participants wrote about their math related feelings $15 \mathrm{~min}$ a day for 3 days. The control group did the same amount of expressive writing but on a neutral topic. The intervention group reported reduced levels of general anxiety and MA, whereas the control group also indicated reduced levels of MA. The students in Ruark (2021) study wrote about their math homework problems every day for 2 weeks. The intervention group was requested to write about their feelings when encountering problems during math homework for at least 1 minute. The control group wrote about their math homework problems only. Both groups showed no reduction of MA.

\subsection{Intervention Mode and Setting}

The interventions were either carried out face-to-face $(67.6 \%)$ or via computer (23.5\%). Three studies (8.8\%) did not fit into one of the two categories. Segumpan and Tan (2018) used both settings-face-to-face and computer-as they investigated the effect of a Flipped Classroom on secondary school students' MA and performance. In Hines et al. (2016) and Ruark (2021) studies the participants performed expressive writing activities at home without specifications whether to use paper and pencil or a computer.

Within the mathematics intervention approach computers were predominantly used to train basic arithmetic operations in primary school children (e.g., Mevarech et al., 1991; Jansen et al., 2013; Huang et al., 2014; Rauscher et al., 2017). Jansen et al. (2013), Rauscher et al. (2017), and Vanbecelaere et al. (2021) explicitly mentioned the adaptivity of their training software, i.e. the selection of training tasks was regulated by an adaptive algorithm (Klinkenberg et al., 2011). The only study within the CBI approach that utilized computers was Kim et al. (2017). In this study secondary school students were guided through a computer-based learning environment by a so-called embodied agent. The learning environment covered fundamental algebra topics. In the intervention group the embodied agent provided not only instructional guidance (control condition) but also anxiety treating messages. Results indicated that both groups obtained lower MA scores and higher math performance. All other CBI were conducted face-to-face.

The interventions were either held in classrooms $(29.4 \%)$, small groups (32.4\%), or individual settings (26.5\%). Four studies (11.8\%) did not specify the setting of their intervention. There were no significant differences between the settings in regards to the intervention approach.

\subsection{Intervention Length}

On average, the included studies applied interventions for $M=$ 7.04 weeks $(S D=6.78)$. However, the span of the overall duration was large. The interventions ranged between a $1-\mathrm{h}$ session (Sheffield and Hunt, 2006) and one school year (Brandenberger and Moser, 2018). Similarly, the number of training sessions varied between the included studies, $M=$ 10.51 sessions $(S D=7.86)$. Again, the span of the number of sessions was large. The interventions took between one session (e.g., Sheffield and Hunt, 2006) and 30 sessions (Rauscher et al., 2017). Accordingly, the number of sessions per week differed, $M=2.6$ sessions/week $(S D=1.4)$. Moreover, the duration of the individual session varied, $M=46.82 \mathrm{~min}(S D=19.85)$, ranging from $15 \mathrm{~min}$ (e.g., Jansen et al., 2013) to $90 \mathrm{~min}$ of intervention time (e.g., Asanjarani and Zarebahramabadi, 2021) in each session.

\subsection{Intervention Effects on MA}

The intervention effects reported by the authors were mixed. 59\% of the studies reported a positive effect of the intervention on MA in the intervention group compared to no effect in the control/ comparison group (e.g., Kramarski et al., 2010; Tok et al., 2015; Alanazi, 2020; Passolunghi et al., 2020). In Passolunghi et al. (2020) study math strategy training influenced and improved not only math ability, but also contributed to a decrease in students' MA level. In the same study the cognitive-behavioral MA training showed only effects in reducing MA level, but there was no improvement of math abilities. Verkijika and De Wet (2015) provided evidence that MA could be effectively reduced by means of neuropsychological feedback while playing a math game. LaGue et al. (2019) reported positive effects of mindfulnessbased cognitive therapy on students' MA levels using an experimental single-case study design.

$21 \%$ of the studies found a positive effect of intervention(s) on MA in both the intervention as well as the control/comparison group (e.g., Jansen et al., 2013; Huang et al., 2014; Hines et al., 2016; Kim et al., 2017; Arias Rodriguez et al., 2019). Rauscher et al. (2017) showed that students who trained with the online math training Calcularis obtained significant lower MA scored compared the waiting list control group (intervention vs. waiting list control group). When compared to the control group that received a control training MA was, however, reduced equally in both groups (intervention vs. control training). Other studies reported a positive effect of the intervention(s) on MA for certain groups of students, such as highly anxious (Supekar et al., 2015; Choi-Koh and Ryoo, 2019) or low achieving students (e.g., Mevarech et al., 1991).

$15 \%$ of the studies did not find a positive effect of the intervention on the students' level of MA (e.g., Shapka and Keating, 2003; Tok, 2013; Collingwood and Dewey, 2018; Vanbecelaere et al., 2020). Collingwood and Dewey (2018) reported a positive impact of intervention on the mathematical performance of students in the intervention group, however, no significant impact on the level of MA. Tok (2013) also found increased achievement after teaching students to use the KnowWant-Learn strategy as well as improved metacognitive abilities, but no significant impact on MA. Shapka and Keating (2003) did not find evidence that girls-only math teaching would reduce female students' MA in comparison to co-educated math teaching. 
The findings did not differ in relation to the applied MA questionnaires. The only study that used a real-time assessment (state-MA) reported a positive effect of a math training on MA, approx. $80 \%$ of the studies using questionnaires with hypothetical/retrospective items (statelike-MA/anxiety in math-related situations) reported lower MA after the intervention and approx. $90 \%$ of the studies using questionnaires focusing anxiety about failure (trait-MA) and anxiety in math-related situations (statelike-MA) reported lower MA after the intervention.

\section{DISCUSSION}

The goal of this study was to summarize the existing body of research on MA interventions for school children. Therefore, we conducted a systematic (scoping) review and presented the results in a narrative manner. Table 1 gives a comprehensive overview of the included studies and their main characteristics. Note that not all studies provided all relevant information.

Generally, the overall number of eligible studies identified in this review was still relatively small, for example compared to general mathematical intervention studies (Reynvoet et al., 2021). Given the potential negative impact of early MA on children's short- and long-term development, one would have expected a greater attention to this field of research. This finding indicates that research on MA interventions is still emerging. The fact that most studies included in this review are relatively recent underpins this assumption. At the same time, the categorization of interventions into either $\mathrm{MI}$ or $\mathrm{CBI}$ as described in adults, can be similarly found in MA research in children and adolescents. The application of both approaches might be justified by different explanations of the MAperformance link (e.g., the reduced competency account and the disruption account of MA; Ramirez et al., 2018). Our findings do not justify any judgments on potential empirical advantages of either approach, as no direct comparisons of the described effects are possible. Future meta-analyses are required to address this issue. At the same time, our findings give qualitative insights into the existing body of research in MA interventions.

More than half of the included studies primarily focused on math performance rather than MA. Hence, MA was often assessed as an affective covariate but was not necessarily the actual target of the intervention. Despite that, almost half of the included MI still reported a positive side-effect of the intervention on students' MA compared to the control/comparison group. This supports the assumption that MI can reduce anxiety responses, but might also allow children to re-evaluate dysfunctional cognitive beliefs ("I am bad at math") and to stimulate the formation of new basic cognitive assumptions (e.g., increase of math self-concept).

As for the CBI, more than half of the included studies reported a positive effect of the intervention on the level of MA compared to the control/comparison group. At the same time, the effect of $\mathrm{CBI}$ on math performance was comparatively low. One possible explanation could be that the physiological arousal that comes with an anxious response (e.g., increased heart rate, faster breathing) can also support performance. Therefore, reducing this arousal through breathing or self-regulation exercises might not always be beneficial to enhance performance. Instead reappraising the arousal as a sign of challenge or excitement rather than threat, might help children to capitalize on the performance enhancing effects of their physiological response see Biopsychological model of Challenge and Threat, (Blascovich, 2008). Similar effects have already been observed in adults (e.g., Brooks, 2014; Jamieson et al., 2016).

The mixed effects of the MI and CBI on MA and performance might indicate that a combination of both approaches could be most beneficial for school children. This means, on the one hand, to develop sound arithmetic skills that build not only the foundation for more complex math content but would also help children to form a positive math self-concept. On the other hand, combined interventions could also provide children with cognitive-behavioral tools to cope with their anxious thoughts and arousal in math related situations. These tools should, however, take effect models into account, such as the Biopsychological model of Challenge and Threat (Blascovich, 2008), that aim to capture the complex interrelations between cognitive processes and affective, physiological, and behavioral responses.

Furthermore, almost a quarter of the described studies, that either apply MI or CBI, reported positive effects on MA for both the intervention and the control/comparison group. This surprising result raises questions on potential third factors that led to a reduction of MA in these studies, and that have not yet been taken explicitly into account. These third factors could be school- and teaching-related variables that might be associated with the development of MA (e.g., teacher's beliefs). At the same time, the differences between the control groups of the included studies hinder potential discussions of these third factor variables. Of course, methodological issues might explain the non-existing differences between control and intervention groups (e.g., nonrandomized controls leading to an unbalanced study design, unknown background interventions). In addition, reductions in the level of MA in both groups might be explained by the applied MA measures. To make differentiated conclusions about impacts of intervention programs on math-related anxiety reactions and/or math anxious cognitive beliefs, it may be useful for future studies to carefully consider the conceptualizations of MA questionnaires. E.g., intervention programs focusing emotional-regulation strategies could benefit from real-time assessments, measuring math-related anxiety reactions (state-MA), whereas studies that incorporate CBI might be more likely to evaluate effects on cognitive beliefs and trait-dispositions. However, to account for all influences, it would be best to consider both situation- and disposition-related approaches.

When comparing the mode and settings of the MI and CBI, it becomes clear that the majority of CBI was based in a one-to-one or small group setting. A classroom-based application of CBI was rare. Hence, future research might try to apply $\mathrm{CBI}$ or to combine CBI and MI on a classroom level. Despite the fact that interventions addressing MA are of relevance for students with 
high levels of MA, all students might profit from adequate strategies targeting anxiety related cognitions.

To conclude, a few limitations of our systematic review need to be mentioned. Firstly, the review only included intervention studies that target MA. This approach might have excluded a range of studies and findings, that highlighted the relevance of potential variables that might also be associated with the development of MA but had not been part of an intervention study (e.g., environmental factors). Secondly, although we tried to capture all relevant information of the included studies as accurate and complete as possible, the transparency within the studies was lacking at times. This implies, that important information might be missing or incomplete for some of the included studies. Especially missing information on the format and duration of the interventions makes it difficult to compare the effectiveness of the different approaches. And thirdly, our review is not a meta-analysis. Insights in described effects are therefore on a descriptive level and do not allow a direct statistical comparison or aggregation of the described effects.

In the end, no clear picture can be drawn yet of how effective MA intervention for school children should look like. However, this literature review still offers valuable insights into the current state in the field of MA intervention research. Both approaches (MI and CBI) show potential positive effects. The findings of this review at hand might therefore serve as an orientation for future

\section{REFERENCES}

Alanazi, H. M. N. (2020). The Effects of Active Recreational Math Games on Math Anxiety and Performance in Primary School Children: An Experimental Study. Mult. J. Edu. Soc. Tec. Sci. 7 (1), 89-112. doi:10. 4995/muse.2020.12622

Alexander, L., and Martray, C. (1989). The Development of an Abbreviated Version of the Mathematics Anxiety Rating Scale. Meas. Eval. Couns. Develop. 22 (3), 143-150. doi:10.1080/07481756.1989.12022923

Arias Rodriguez, I., Mendes do Nascimento, J., Voigt, M. F., and Dos Santos, F. H. (2019). Numeracy Musical Training for School Children with Low Achievement in Mathematics. An Psicol-Spain 35 (3), 405-416. doi:10.6018/ analesps.35.3.340091

Asanjarani, F., and Zarebahramabadi, M. (2021). Evaluating the Effectiveness of Cognitive-Behavioral Therapy on Math Self-Concept and Math Anxiety of Elementary School Students. Preventing Sch. Fail. Altern. Edu. Child. Youth 65 (3), 223-229. doi:10.1080/1045988X.2021.1888685

Ashcraft, M. H., and Moore, A. M. (2009). Mathematics Anxiety and the Affective Drop in Performance. J. Psychoeducational Assess. 27 (3), 197-205. doi:10.1177/ 0734282908330580

Asikhia, O. A., and Mohangi, K. (2015). The Use of Problem-Solving Training in Reducing Mathematics Anxiety Among Nigerian Secondary School Students. Gend. Behav. 13 (1), 6547-6558.

Barroso, C., Ganley, C. M., McGraw, A. L., Geer, E. A., Hart, S. A., and Daucourt, M. C. (2021). A Meta-Analysis of the Relation between Math Anxiety and Math Achievement. Psychol. Bull. 147 (2), 134-168. doi:10. 1037/bul0000307

Bieg, M. (2013). Trait And State Academic Emotions: Two Sides Of the Same Coin. Dissertation. Germany: University of Konstanz. Available: https://kops.unikonstanz.de/handle/123456789/25394 (Accessed January 14, 2022). research and for the development of effective interventions that aim to reduce MA in children.

\section{DATA AVAILABILITY STATEMENT}

The raw data supporting the conclusion of this article will be made available by the authors on request, without undue reservation.

\section{AUTHOR CONTRIBUTIONS}

LO, MB and MB-R drafted the theoretical background. $\mathrm{MB}$ and MB-R were responsible for data analysis and discussion of the findings. All authors contributed to the article and approved the submitted version.

\section{ACKNOWLEDGMENTS}

We thank Julia Gehlhaus, Lisa Marie Flebbe, and Kristin Busse for their support in screening and evaluating the studies for this systematic review and for piloting the data extraction spread sheet as part of their Bachelor theses. We acknowledge support from the Open Access Publication Fund of the University of Wuppertal.

Bindak, R. (2005). İlköğretim Öğrencileri Için Matematik Kaygı̈̈lçeği [Math Anxiety Scale for Elementary School Students]. F.Ü. Fen Ve Mühendislik Bilimleri Dergisi 17 (2), 442-448.

Blascovich, J. (2008). "Challenge and Threat," in Handbook of Approach and Avoidance Motivation. Editor A. J. Elliot (New York: Psychology Press), 431-445.

Brandenberger, C. C., and Moser, N. (2018). "Förderung der Lernfreude und Reduzierung der Angst im Mathematikunterricht in der Sekundarstufe 1 [Supporting joy of learning and reducing anxiety in secondary school mathematics classrooms," in Emotionen und Emotionsregulation in der Schule und Hochschule. Editors G. Hagenauer, and T. Hascher (Münster: Waxmann).

Brooks, A. W. (2014). Get Excited: Reappraising Pre-performance Anxiety as Excitement. J. Exp. Psychol. Gen. 143 (3), 1144-1158. doi:10.1037/a0035325

Carey, E., Hill, F., Devine, A., and Szücs, D. (2016). The Chicken or the Egg? The Direction of the Relationship between Mathematics Anxiety and Mathematics Performance. Front. Psychol. 6, 1987. doi:10.3389/fpsyg.2015.01987

Carey, E., Hill, F., Devine, A., and Szücs, D. (2017). The Modified Abbreviated Math Anxiety Scale: A Valid and Reliable Instrument for Use with Children. Front. Psychol. 8, 11. doi:10.3389/fpsyg.2017.00011

Cargnelutti, E., Tomasetto, C., and Passolunghi, M. C. (2017). How Is Anxiety Related to Math Performance in Young Students? A Longitudinal Study of Grade 2 to Grade 3 Children. Cogn. Emot. 31 (4), 755-764. doi:10.1080/ 02699931.2016 .1147421

Carmo, J. S. (2008). Escala de Ansiedade à Matemática [Mathematics Anxiety Scale]. Unpublished Manuscript. Brazil: Federal University of São Carlos.

Casad, B. J., Hale, P., and Wachs, F. L. (2015). Parent-Child Math Anxiety and Math-Gender Stereotypes Predict Adolescents' Math Education Outcomes. Front. Psychol. 6, 1597. doi:10.3389/fpsyg.2015.01597

Cavanagh, R., and Sparrow, L. (2011). "Mathematics Anxiety: Scafolding a New Construct Model," in Mathematics Education Research Group Australasia 
(MERGA) Conference Proceedings, Alice Springs, Australia, July 3-7, 2011, 166-173. Available at:https://www.merga.net.au/Public/Public/Publications/ Annual_Conference_Proceedings/2011_MERGA_CP.aspx (Accessed January 14, 2022).

Caviola, S., Carey, E., Mammarella, I. C., and Szucs, D. (2017a). Stress, Time Pressure, Strategy Selection and Math Anxiety in Mathematics: A Review of the Literature. Front. Psychol. 8, 1488. doi:10.3389/fpsyg.2017.01488

Caviola, S., Primi, C., Chiesi, F., and Mammarella, I. C. (2017b). Psychometric Properties of the Abbreviated Math Anxiety Scale (AMAS) in Italian Primary School Children. Learn. Individual Differences 55, 174-182. doi:10.1016/j.lindif. 2017.03.006

Chiu, L. -H., and Henry, L. L. (1990). Development and Validation of the Mathematics Anxiety Scale for Children. Meas. Eval. Couns. Develop. 23 (3), $121-127$.

Choi-Koh, S. S., and Ryoo, B. G. (2019). Differences of Math Anxiety Groups Based on Two Measurements, MASS and EEG. Educ. Psychol. 39 (5), 659-677. doi:10. $1080 / 01443410.2018 .1543857$

Collingwood, N., and Dewey, J. (2018). Thinking Your Problems Away': Can Maths Interventions Be Developed to Address Both the Academic and Affective Aspects of Learning in Primary Aged Children? Educ. Child Psychol. 35 (2), 76-92.

Da Silva, E. R., Dos Santos Baldin, M., and Dos Santos, F. H. (2017). Cognitive Effects of Numeracy Musical Training in Brazilian Preschool Children: A Prospective Pilot Study. Psychol. Neurosci. 10 (3), 281-296. doi:10.1037/ pne0000098

Dowker, A., Sarkar, A., and Looi, C. Y. (2016). Mathematics Anxiety: What Have We Learned in 60 years? Front. Psychol. 7, 508. doi:10.3389/fpsyg.2016.00508

Ellis, A., and Bernard, M. E. (Editors) (2006). Rational-Emotive Behavioral Approaches to Childhood Disorders: Theory, Practice and Research. Boston, MA: Springer Science \& Business Media.

Foley, A. E., Herts, J. B., Borgonovi, F., Guerriero, S., Levine, S. C., and Beilock, S. L. (2017). The Math Anxiety-Performance Link. Curr. Dir. Psychol. Sci. 26 (1), 52-58. doi:10.1177/0963721416672463

Fuchs, L. S., Fuchs, D., Schumacher, R. F., and Seethaler, P. M. (2013). "Instructional Intervention for Students with Mathematics Learning Disabilities," in Handbook of Learning Disabilities. Editors H. L. Swanson, K. R. Harris, and S. Graham (New York: The Guilford Press), 388-404.

Gunderson, E. A., Park, D., Maloney, E. A., Beilock, S. L., and Levine, S. C. (2018). Reciprocal Relations Among Motivational Frameworks, Math Anxiety, and Math Achievement in Early Elementary School. J. Cogn. Develop. 19 (1), 21-46. doi:10.1080/15248372.2017.1421538

Hativa, N., Shapira, R., and Navon, D. (1990). Computer-Managed Practice: Effects on Instructional Methods and on Teacher Adoption. Teach. Teach. Edu. 6 (1), 55-68. doi:10.1016/0742-051x(90)90007-r

Hines, C. L., Brown, N. W., and Myran, S. (2016). The Effects of Expressive Writing on General and Mathematics Anxiety for a Sample of High School Students. Education 137 (1), 39-45.

Hord, C., Marita, S., Ayaz, S., Tomaro, T. M., Gordon, K., Tunningley, J., et al. (2018). Diverse Needs of Students with Learning Disabilities: A Case Study of Tutoring Two Students in Algebra. J. Res. Spec. Educ. Needs 18 (Suppl. 1), 25-35. doi:10.1111/1471-3802.12415

Huang, Y.-M., Huang, S.-H., and Wu, T.-T. (2014). Embedding Diagnostic Mechanisms in a Digital Game for Learning Mathematics. Education Tech Res. Dev 62 (2), 187-207. doi:10.1007/s11423-013-9315-4

Idris, N. (2006). Exploring the Effects of TI-84 Plus on Achievement and Anxiety in Mathematics. Eurasia J. Math. Sci. Tech. Edu. 2 (3), 66-78. doi:10.12973/ejmste/ 75465

Jamieson, J. P., Peters, B. J., Greenwood, E. J., and Altose, A. J. (2016). Reappraising Stress Arousal Improves Performance and Reduces Evaluation Anxiety in Classroom Exam Situations. Soc. Psychol. Personal. Sci. 7 (6), 579-587. doi:10.1177/1948550616644656

Jansen, B. R. J., Louwerse, J., Straatemeier, M., Van der Ven, S. H. G., Klinkenberg, S., and Van der Maas, H. L. J. (2013). The Influence of Experiencing success in Math on Math Anxiety, Perceived Math Competence, and Math Performance. Learn. Individual Differences 24, 190-197. doi:10.1016/j.lindif. 2012.12.014
Käser, T., Baschera, G. M., Kohn, J., Kucian, K., Richtmann, V., Grond, U., et al. (2013). Design and Evaluation of the Computer-Based Training Program Calcularis for Enhancing Numerical Cognition. Front. Psychol. 4, 489-513. doi:10.3389/fpsyg.2013.00489

Kamann, M. P., and Wong, B. Y. (1993). Inducing Adaptive Coping SelfStatements in Children with Learning Disabilities through Self-Instruction Training. J. Learn. Disabil. 26 (9), 630-638. doi:10.1177/002221949302600913

Karimi, A., and Venkatesan, S. (2009). Cognitive Behavior Group Therapy in Mathematics Anxiety. J. Indian Acad. Appl. Psychol. 35 (2), 299-303.

Kazelskis, R., and Reeves, C. (2002). The Fennema-Sherman Mathematics Anxiety Scale: An Exploratory Factor Analysis. Res. Schools 9 (1), 61-64.

Kim, Y., Thayne, J., and Wei, Q. (2017). An Embodied Agent Helps Anxious Students in Mathematics Learning. Edu. Tech Res. Dev 65 (1), 219-235. doi:10. 1007/s11423-016-9476-z

Kim, E. J. (2010). A Study on the Development of a Brain Education Program to Improve Self Directed Learning Ability. Korean J. Youth Stud. 17 (10), 161-188.

Klinkenberg, S., Straatemeier, M., and van der Maas, H. L. J. (2011). Computer Adaptive Practice of Maths Ability Using a New Item Response Model for on the Fly Ability and Difficulty Estimation. Comput. Edu. 57, 1813-1824. doi:10. 1016/j.compedu.2011.02.003

Ko, H. K., and Yi, H. S. (2011). Development and Validation of a Mathematics Anxiety Scale for Students. Asia Pac. Educ. Rev. 12 (4), 509-521. doi:10.1007/ s12564-011-9150-4

Kohn, J., Richtmann, V., Rauscher, L., Kucian, K., Käser, T., Grond, U., et al. (2013). Das Mathematikangstinterview (MAI): Erste Psychometrische Gütekriterien. Lernen und Lernstörungen 2 (3), 177-189. doi:10.1024/22350977/a000040

Kramarski, B., Weisse, I., and Kololshi-Minsker, I. (2010). How Can Self-Regulated Learning Support the Problem Solving of Third-Grade Students with Mathematics Anxiety? ZDM Math. Edu. 42 (2), 179-193. doi:10.1007/ s11858-009-0202-8

LaGue, A., Eakin, G., and Dykeman, C. (2019). The Impact of Mindfulness-Based Cognitive Therapy on Math Anxiety in Adolescents. Preventing Sch. Fail. Altern. Edu. Child. Youth 63 (2), 142-148. doi:10.1080/1045988X.2018.1528966

Lavasani, M. G., Hejazi, E., and Khandan, F. (2012). The Effect of Cooperative Learning on Mathematics Anxiety and Help Seeking Behavior. J. Psychol. 15 (4), 397-411.

Lin, Y., Durbin, J. M., and Rancer, A. S. (2017). Perceived Instructor Argumentativeness, Verbal Aggressiveness, and Classroom Communication Climate in Relation to Student State Motivation and Math Anxiety. Commun. Edu. 66 (3), 330-349. doi:10.1080/03634523.2016.1245427

Maertens, B., De Smedt, B., Sasanguie, D., Elen, J., and Reynvoet, B. (2016). Enhancing Arithmetic in Pre-Schoolers with Comparison or Number Line Estimation Training: Does it Matter? Learn. Instruction 46, 1-11. doi:10.1016/j. learninstruc.2016.08.004

Malanchini, M., Rimfeld, K., Shakeshaft, N. G., Rodic, M., Schofield, K., Selzam, S., et al. (2017). The Genetic and Environmental Aetiology of Spatial, Mathematics and General Anxiety. Sci. Rep. 7 (1), 42218-42311. doi:10.1038/srep42218

Martin, A. J. (2008). Enhancing Student Motivation and Engagement: The Effects of a Multidimensional Intervention. Contemp. Educ. Psychol. 33 (2), 239-269. doi:10.1016/j.cedpsych.2006.11.003

May, D. K. (2009). Mathematics Self-Efficacy and Anxiety Questionnaire. Dissertation. Athens: University of Georgia. Available at: https://getd.libs. uga.edu/pdfs/may_diana_k_200908_phd.pdf (Accessed January 14, 2022).

Mehdizadeh, S., Nojabaee, S. S., and Asgari, M. H. (2013). The Effect of Cooperative Learning on Math Anxiety, Help Seeking Behavior. J. Basic Appl. Scientific Res. 3 (3), 1185-1190.

Meichenbaum, D. (1977). Cognitive Behaviour Modification: An Integrative Approach. New York: Plenum Press.

Mevarech, Z. R., and Kramarski, B. (1997). Improve: A Multidimensional Method for Teaching Mathematics in Heterogeneous Classrooms. Am. Educ. Res. J. 34 (2), 365-394. doi:10.3102/00028312034002365

Mevarech, Z. R., and Rich, Y. (1985). Effects of Computer-Assisted Mathematics Instruction on Disadvantaged Pupils' Cognitive and Affective Development. J. Educ. Res. 79 (1), 5-11. doi:10.1080/00220671.1985.10885638 
Mevarech, Z. R., Silber, O., and Fine, D. (1991). Learning with Computers in Small Groups: Cognitive and Affective Outcomes. J. Educ. Comput. Res. 7 (2), 233-243. doi:10.2190/BRCJ-P9K5-9E1D-2ALC

Midgley, C., Kaplan, A., Middleton, M., Maehr, M. L., Urdan, T., Anderman, L. H., et al. (2013). Manual for the Patterns of Adaptive Learning Scales. Ann Arbor: University of Michigan.

Miller, P. H., and Seier, W. L. (1994). Strategy Utilization Deficiencies in Children: When, where, and Why. Adv. child Dev. Behav. 25, 107-156. Academic Press. doi:10.1016/S0065-2407(08)60051-8

Moher, D., Liberati, A., Tetzlaff, J., and Altman, D. G. (2009). Preferred Reporting Items for Systematic Reviews and Meta-Analyses: The PRISMA Statement. BMJ 339 (7), b2535-b2536. doi:10.1136/bmj.b2535

Namkung, J. M., Peng, P., and Lin, X. (2019). The Relation between Mathematics Anxiety and Mathematics Performance Among School-Aged Students: A MetaAnalysis. Rev. Educ. Res. 89 (3), 459-496. doi:10.3102/0034654319843494

OECD (2005). PISA 2003 Technical Report. Paris: OECD Publishing. doi:10.1787/ 9789264010543-en

Orbach, L., Herzog, M., and Fritz, A. (2019). Relation of State- and Trait-Math Anxiety to Intelligence, Math Achievement and Learning Motivation. J. Numer. Cogn. 5 (3), 371-399. doi:10.5964/jnc.v5i3.204

Orbach, L., Herzog, M., and Fritz, A. (2020). Relation of Attention Deficit Hyperactivity Disorder (ADHD) to Basic Number Skills and Arithmetic Fact Retrieval in Children. Res. Dev. Disabil. 103, 103697. doi:10.1016/j.ridd. 2020.103697

Ouzzani, M., Hammady, H., Fedorowicz, Z., and Elmagarmid, A. (2016). Rayyan-a Web and mobile App for Systematic Reviews. Syst. Rev. 5 (210), 210-10. doi:10. 1186/s13643-016-0384-4

Passolunghi, M. C., De Vita, C., and Pellizzoni, S. (2020). Math Anxiety and Math Achievement: The Effects of Emotional and Math Strategy Training. Dev. Sci. 23, e12964. doi:10.1111/desc.12964

Pekrun, R., Goetz, T., and Frenzel, A. C. (2005). Academic Emotions Questionnaire-Mathematics (AEQ-M): User's Manual. Munich: University of Munich, Department of Psychology.

Pizzie, R. G., Raman, N., and Kraemer, D. J. M. (2020). Math Anxiety and Executive Function: Neural Influences of Task Switching on Arithmetic Processing. Cogn. Affect Behav. Neurosci. 20 (2), 309-325. doi:10.3758/s13415-020-00770-z

Plake, B. S., and Parker, C. S. (1982). The Development and Validation of a Revised Version of the Mathematics Anxiety Rating Scale. Educ. Psychol. Meas. 42 (2), 551-557. doi:10.1177/001316448204200218

Primi, C., Donati, M. A., Izzo, V. A., Guardabassi, V., O'Connor, P. A., Tomasetto, C., et al. (2020). The Early Elementary School Abbreviated Math Anxiety Scale (The EES-AMAS): A New Adapted Version of the AMAS to Measure Math Anxiety in Young Children. Front. Psychol. 11, 1014. doi:10.3389/fpsyg.2020. 01014

Ramirez, G., Gunderson, E. A., Levine, S. C., and Beilock, S. L. (2013). Math Anxiety, Working Memory, and Math Achievement in Early Elementary School. J. Cogn. Develop. 14 (2), 187-202. doi:10.1080/15248372.2012.664593

Ramirez, G., Chang, H., Maloney, E. A., Levine, S. C., and Beilock, S. L. (2016). On the Relationship between Math Anxiety and Math Achievement in Early Elementary School: The Role of Problem Solving Strategies. J. Exp. Child. Psychol. 141, 83-100. doi:10.1016/j.jecp.2015.07.014

Ramirez, G., Shaw, S. T., and Maloney, E. A. (2018). Math Anxiety: Past Research, Promising Interventions, and a New Interpretation Framework. Educ. Psychol. 53 (3), 145-164. doi:10.1080/00461520.2018.1447384

Rauscher, L., Kohn, J., Käser, T., Kucian, K., McCaskey, U., Wyschkon, A., et al. (2017). Effekte des Calcularis-Trainings. Lernen und Lernstörungen 6 (2), 75-86. doi:10.1024/2235-0977/a000168

Reynvoet, B., Vanbecelaere, S., Depaepe, F., and Sasanguie, D. (2021). "Intervention Studies in Math: A Metareview," in Heterogeneous Contributions to Numerical Cognition. Editors W. Fias and A. Hanik (Cambridge, MA: Academic Press), 283-308. doi:10.1016/b978-0-12-8174142.00012-9

Ruark, A. (2021). Expressive Writing as an Intervention for Math Anxiety in Middle School Students. MLET: J. Middle Level Edu. Tex. 7 (1), 1.

Ruff, S. E., and Boes, S. R. (2014). The Sum of All Fears: The Effects of Math Anxiety on Math Achievement in Fifth Grade Students and the Implications for School Counselors. Ga. Sch. Counselors Assoc. J. 21 (1).
Sarason, I. G. (1980). Test Anxiety: Theory, Research, and Applications. Hillsdale: Lawrence Erlbaum.

Sarason, I. G. (1986). "Test Anxiety, Worry, and Cognitive Interference," in SelfRelated Cognitions in Anxiety and Motivation. Editor R. Schwarzer (Hillslade: Lawrence Erlbaum), 19-35.

Segumpan, L. L. B., and Tan, D. A. (2018). Mathematics Performance and Anxiety of Junior High School Students in a Flipped Classroom. Eur. J. Educ. Stud. 4 (12), 1-33. doi:10.5281/ZENODO.1325918

Shapka, J. D., and Keating, D. P. (2003). Effects of a Girls-Only Curriculum during Adolescence: Performance, Persistence, and Engagement in Mathematics and Science. Am. Educ. Res. J. 40 (4), 929-960. doi:10.3102/00028312040004929

Sheffield, D., and Hunt, T. (2006). How Does Anxiety Influence Maths Performance and what Can We Do about it? MSOR Connections 6 (4), 19-23. doi:10.11120/msor.2006.06040019

Shie, B.-H. (2006). Construction of Mathematics Anxiety Scale for Younger Pupils: Verification of Theoretical Models. Unpublished Master Thesis. Taipei: National Taipei University of Education.

Shokrani, M. (2002). Creation and Validity Recognition of Math Anxiety Scale of Measurement at Secondary School Students in Khomeini Shahr and Investigation of Factors Related to Math Anxiety. Unpublished Master Thesis. Tehran: University of Tehran.

Singh, P. (2016). Management of Mathematics Phobia Among Ninth Standard Students. Int. J. Indian Psychol. 3 (2), 69-76. doi:10.25215/0302.043

Sorvo, R., Koponen, T., Viholainen, H., Aro, T., Räikkönen, E., Peura, P., et al. (2017). Math Anxiety and its Relationship with Basic Arithmetic Skills Among Primary School Children. Br. J. Educ. Psychol. 87 (3), 309-327. doi:10.1111/bjep.12151

Sorvo, R., Koponen, T., Viholainen, H., Aro, T., Räikkönen, E., Peura, P., et al. (2019). Development of Math Anxiety and its Longitudinal Relationships with Arithmetic Achievement Among Primary School Children. Learn. Individual Differences 69, 173-181. doi:10.1016/j.lindif.2018.12.005

Spielberger, C. D. (1972). Anxiety. Current Trends in Theory and Research. New York: Academic Press.

Suinn, R. M., Edie, C. A., Nicoletti, J., and Spinelli, P. R. (1972). The MARS, a Measure of Mathematics Anxiety: Psychometric Data. J. Clin. Psychol. 28 (6), 373-375. doi:10.1002/1097-4679(197207)28:3+<373:aid-jclp2270280320>3. $0 . \operatorname{co} ; 2-\mathrm{c}$

Suinn, R. M., and Edwards, R. (1982). The Measurement of Mathematics Anxiety: The Mathematics Anxiety Rating Scale for Adolescents--MARS-A. J. Clin. Psychol. 38 (3), 576-580. doi:10.1002/1097-4679(198207)38:3<576:aidjclp2270380317>3.0.co;2-v

Suinn, R. M., and Winston, E. H. (2003). The Mathematics Anxiety Rating Scale, a Brief Version: Psychometric Data. Psychol. Rep. 92 (1), 167-173. doi:10.2466/ pr0.2003.92.1.167

Supekar, K., Iuculano, T., Chen, L., and Menon, V. (2015). Remediation of Childhood Math Anxiety and Associated Neural Circuits through Cognitive Tutoring. J. Neurosci. 35 (36), 12574-12583. doi:10.1523/JNEUROSCI.0786-15.2015

Tok, Ş., Bahtiyar, A., and Karalök, S. (2015). The Effects of Teaching Mathematics Creatively on Academic Achievement, Attitudes towards Mathematics, and Mathematics Anxiety. Int. J. Innovation Sci. Math. Edu. 23 (4), 1-24.

Tok, S. (2013). Effects of the Know-Want-Learn Strategy on Students' Mathematics Achievement, Anxiety and Metacognitive Skills. Metacognition Learn. 8 (2), 193-212. doi:10.1007/s11409-013-9101-z

Vanbecelaere, S., Van den Berghe, K., Cornillie, F., Sasanguie, D., Reynvoet, B., and Depaepe, F. (2020). The Effects of Two Digital Educational Games on Cognitive and Non-Cognitive Math and reading Outcomes. Comput. Edu. 143, 103680. doi:10.1016/j.compedu.2019.103680

Vanbecelaere, S., Cornillie, F., Sasanguie, D., Reynvoet, B., and Depaepe, F. (2021). The Effectiveness of an Adaptive Digital Educational Game for the Training of Early Numerical Abilities in Terms of Cognitive, Noncognitive and Efficiency Outcomes. Br. J. Educ. Technol. 52 (1), 112-124. doi:10.1111/bjet.12957

Verkijika, S. F., and De Wet, L. (2015). Using a Brain-Computer Interface (BCI) in Reducing Math Anxiety: Evidence from South Africa. Comput. Edu. 81, 113-122. doi:10.1016/j.compedu.2014.10.002

Wang, Z., Hart, S. A., Kovas, Y., Lukowski, S., Soden, B., Thompson, L. A., et al. (2014). Who Is Afraid of Math? Two Sources of Genetic Variance for Mathematical Anxiety. J. Child. Psychol. Psychiatry 55 (9), 1056-1064. doi:10.1111/jcpp.12224 
Wu, S. S., Barth, M., Amin, H., Malcarne, V., and Menon, V. (2012). Math Anxiety in Second and Third Graders and its Relation to Mathematics Achievement. Front. Psychol. 3 (162), 162. doi:10.3389/fpsyg.2012.00162

Young, C. B., Wu, S. S., and Menon, V. (2012). The Neurodevelopmental Basis of Math Anxiety. Psychol. Sci. 23 (5), 492-501. doi:10.1177/ 0956797611429134

Zhang, J., Zhao, N., and Kong, Q. P. (2019). The Relationship between Math Anxiety and Math Performance: A Meta-Analytic Investigation. Front. Psychol. 10, 1613. doi:10.3389/fpsyg.2019.01613

Conflict of Interest: The authors declare that the research was conducted in the absence of any commercial or financial relationships that could be construed as a potential conflict of interest.
Publisher's Note: All claims expressed in this article are solely those of the authors and do not necessarily represent those of their affiliated organizations, or those of the publisher, the editors, and the reviewers. Any product that may be evaluated in this article, or claim that may be made by its manufacturer, is not guaranteed or endorsed by the publisher.

Copyright (๑) 2022 Balt, Börnert-Ringleb and Orbach. This is an open-access article distributed under the terms of the Creative Commons Attribution License (CC BY).

The use, distribution or reproduction in other forums is permitted, provided the original author(s) and the copyright owner(s) are credited and that the original publication in this journal is cited, in accordance with accepted academic practice. No use, distribution or reproduction is permitted which does not comply with these terms. 